$11-1-2012$

\title{
Bayesian Estimation of Erlang Distribution under Different Generalized Truncated Distributions as Priors
}

\author{
Adil H. Khan \\ University of Kashmir, Srinagar, India \\ T.R. Jan \\ University of Kashmir, Srinagar, India
}

Follow this and additional works at: http://digitalcommons.wayne.edu/jmasm

Part of the Applied Statistics Commons, Social and Behavioral Sciences Commons, and the Statistical Theory Commons

\section{Recommended Citation}

Khan, Adil H. and Jan, T.R. (2012) "Bayesian Estimation of Erlang Distribution under Different Generalized Truncated Distributions as Priors," Journal of Modern Applied Statistical Methods: Vol. 11 : Iss. 2 , Article 14.

DOI: $10.22237 /$ jmasm/1351743180

Available at: http://digitalcommons.wayne.edu/jmasm/vol11/iss2/14

This Regular Article is brought to you for free and open access by the Open Access Journals at DigitalCommons@WayneState. It has been accepted for inclusion in Journal of Modern Applied Statistical Methods by an authorized editor of DigitalCommons@WayneState. 


\section{Bayesian Estimation of Erlang Distribution under Different Generalized Truncated Distributions as Priors}

\section{Cover Page Footnote}

The authors would like to thanks the referees for their careful reading of the manuscript and for their valuable comments and suggestions. 


\title{
Bayesian Estimation of Erlang Distribution under Different Generalized Truncated Distributions as Priors
}

\author{
Adil H. Khan T. R. Jan \\ University of Kashmir, \\ Srinagar. India
}

Various generalized truncated distributions are considered as independent informative priors for estimating shape and scale parameters of the Erlang distribution. In addition, various special cases are also discussed.

Key words: Erlang distribution, generalized truncated distributions, Bayes estimator, posterior distribution.

\section{Introduction}

The Erlang distribution is a continuous probability distribution with wide applicability, primarily due to its relation to the exponential and Gamma distributions. The Erlang distribution was developed by A. K. Erlang (1909) to examine the number of telephone calls that could be made at the same time to switching station operators. This work on telephone traffic engineering has been expanded to consider waiting times in queuing systems in general. Queuing theory originated when Erlang (1909) published his fundamental paper relating to the study of telephone traffic congestion (Brockmeyer, Halstorm \& Jenson, 1948).

The probability function of the Erlang distribution is

$$
\begin{aligned}
& f(x: u, v)=\frac{x^{u-1} \exp \left(-v^{-1} x\right)}{\Gamma(u) v^{u}} ; \\
& u=1,2,3, \ldots ; v>0, x>0
\end{aligned}
$$

Adil H. Khan is a Research Scholar in the PostGraduate Department of Statistics. Email him at: khanadil_192@yahoo.com. T. R. Jan is an Assistant Professor in the Post-Graduate Department of Statistics, University of Kashmir Email him at: drtrjan@gmail.com. where $\mathrm{u}$ and $\mathrm{v}$ are unknown parameters and are respectively called shape and scale parameters. When the scale parameter $\mathrm{v}=2$, the distribution simplifies to a Chi-squared distribution with $2 \mathrm{k}$ degrees of freedom; therefore, it can be regarded as a generalized Chi-squared distribution.

The Erlang distribution is the distribution of $u$ independent identically distributed random variables each with an exponential distribution, and can be expressed as waiting time and message length in telephone traffic. If the durations of individual calls are exponentially distributed, then the duration of successive calls is the Erlang distribution. The Erlang distribution is a special case of the Gamma distribution when the shape parameter $\mathrm{u}$ is an integer (Evans, et al., 2000).

Harischandra and Rao (1998) discussed problems with classical inferences for the Erlangian queue. Bhattacharyya and Singh (1994) obtained a Bayes estimator for the Erlangian queue under two prior densities. Wiper (1998) studied $\mathrm{Er} / \mathrm{M} / 1$ and $\mathrm{Er} / \mathrm{M} / \mathrm{C}$ queues under a Bayesian setup and estimated equilibrium probabilities of queue size and waiting time distribution using conditional Monte-Carlo simulation methods. Jain (2001) examined the problem of change point for the inter-arrival time distribution in the context of exponential families for the Ek/G/e queuing system and obtained a Bayes estimate of posterior probabilities and the position of the change from the Erlang distribution. Nair, et al. 
(2003) studied the Erlang distribution as a model for ocean wave periods and obtained different characteristics of the distribution under a classical setup. Suri, et al (2009) used the Erlang distribution to design a simulator for project management process time estimation. Recently, Damodaran, et al. (2010) obtained the expected time between failure measures and showed that the predicted failure times are closer to the actual failure time. Haq and Dey (2011) addressed the problem of Bayesian estimation of parameters for the Erlang distribution assuming different independent informative priors. This article estimates parameters of the Erlang distribution using different generalized truncated distributions.

\section{Prior and Loss Functions}

In many practical situations information is available regarding the shape and scale parameters of sampling distributions, therefore, this article considers a number of prior distributions and assumes that that the parameters $\mathrm{u}$ and $\mathrm{v}$ are independent. The distributions considered, which are priors for shape and scale parameters, are:

(a) Generalized Truncated Poisson;

(b) Generalized Truncated Geometric;

(c) Generalized Truncated Poisson and Inverted Gamma;

(d) Generalized Truncated Poisson and Gamma;

(e) Generalized Truncated Geometric and Inverted Gamma; and the

(f) Generalized Truncated Geometric and Gamma.

The loss function considered is a squared error loss function. The squared error loss function for the shape parameters (u) and the scale parameters (v) are defined as

$$
\mathrm{L}(\hat{\mathrm{u}})=(\hat{\mathrm{u}}-\mathrm{u})^{2}
$$

and

$$
\mathrm{L}(\hat{\mathrm{v}})=(\hat{\mathrm{v}}-\mathrm{v})^{2}
$$

which are symmetric, and where $\mathrm{u}$ and $\mathrm{v}$, and $\hat{\mathrm{u}}$ and $\hat{\mathrm{v}}$ represent the true and estimated values of the parameters.

Derivation of Posterior Distribution under Different Informative Priors

If $X_{1}, X_{2}, X_{3}, \ldots, X_{n}$ are a random sample from the Erlang distribution, then the likelihood function of sample observations $\mathrm{x}_{1}$, $\mathrm{x}_{2}, \mathrm{x}_{3}, \ldots, \mathrm{x}_{\mathrm{n}}$ is defined as:

$$
\begin{aligned}
& \mathrm{L}(\mathrm{u}, \mathrm{v}: \mathrm{x})=\frac{\prod_{\mathrm{i}=1}^{\mathrm{n}} \mathrm{x}_{\mathrm{i}} \exp \left(-\mathrm{v} \sum_{\mathrm{i}=1}^{\mathrm{n}} \mathrm{x}_{\mathrm{i}}\right)}{(\Gamma(\mathrm{u}))^{\mathrm{n}} \mathrm{v}^{\mathrm{nu}}} ; \\
& \mathrm{u}=1,2,3, \ldots ; \mathrm{v}>0
\end{aligned}
$$

When shape parameter $\mathrm{u}$ is unknown and scale parameter $\mathrm{v}$ is known, then the performance of the Bayes estimators depends on the form of the prior distribution and the loss function assumed. Two different informative prior distributions are assumed for the shape parameter $\mathrm{u}$, namely, the generalized truncated Poisson distribution and the generalized truncated geometric distribution. These are used to obtain the Bayes estimators and posterior variances. Also, Bayes estimators and posterior variances are also obtained for the truncated Poisson distribution and the truncated geometric distribution as the special cases.

Generalized Truncated Poisson Distribution as a Prior for Shape Parameter u

The probability density function (pdf) of the generalized truncated Poisson distribution is:

$$
\begin{aligned}
& \mathrm{g}_{1}\left(\mathrm{u}_{1}, \theta_{1}\right)=\frac{(1+\alpha \mathrm{u})^{\mathrm{u}-1} \theta_{1}^{\mathrm{u}} \exp \left(-\theta_{1}(1+\alpha \mathrm{u})\right)}{\Gamma(\mathrm{u}+1)\left(1-\exp \left(-\theta_{1}\right)\right)} ; \\
& \mathrm{u}=1,2,3, \ldots, \theta_{1}>0 .
\end{aligned}
$$

For $\alpha=0$ the truncated Poisson distribution is: 


$$
\begin{aligned}
& \mathrm{g}_{1}^{*}\left(\mathrm{u}_{1}, \theta_{1}\right)=\frac{\theta_{1}^{\mathrm{u}} \exp \left(-\theta_{1}\right)}{\Gamma(\mathrm{u}+1)\left(1-\exp \left(-\theta_{1}\right)\right)} \\
& \mathrm{u}=1,2,3, \ldots, \theta_{1}>0 .
\end{aligned}
$$

By combining likelihood function (3.1) with prior density function (4.1) the posterior distribution of $\mathrm{u}$, the prior is the generalized truncated Poisson distribution:

$\mathrm{g}_{1}(\mathrm{u} / \mathrm{x})=$

$\frac{\frac{(1+\alpha u)^{\mathrm{u}-1} \theta_{1}^{\mathrm{u}} \exp \left(-\theta_{1}(1+\alpha \mathrm{u})\right) \exp \left(\mathrm{u} \sum_{\mathrm{i}=1}^{\mathrm{n}} \ln \mathrm{x}_{\mathrm{i}}\right.}{\Gamma(\mathrm{u}+1)(\Gamma(\mathrm{u}))^{\mathrm{n}} \mathrm{v}^{\mathrm{nu}}}}{\sum_{\mathrm{u}=1}^{\infty}\left(\frac{(1+\alpha \mathrm{u})^{\mathrm{u}-1} \theta_{1}^{\mathrm{u}} \exp \left(-\theta_{1}(1+\alpha \mathrm{u})\right) \exp \left(\mathrm{u} \sum_{\mathrm{i}=1}^{\mathrm{n}} \ln \mathrm{x}_{\mathrm{i}}\right.}{\Gamma(\mathrm{u}+1)(\Gamma(\mathrm{u}))^{\mathrm{n}} \mathrm{v}^{\mathrm{nu}}}\right)} ;$

$\mathrm{u}=1,2, \ldots$

Under the squared error loss function with the prior $\mathrm{g}_{1}\left(\mathrm{u}_{1}, \theta_{1}\right)$, the Bayes estimator is

$$
\begin{aligned}
& \hat{\mathrm{u}}_{1} / \mathrm{X}= \\
& \frac{\sum_{\mathrm{u}=1}^{\infty}\left(\frac{\mathrm{u}(1+\alpha \mathrm{u})^{\mathrm{u}-1} \theta_{1}^{\mathrm{u}} \exp \left(-\theta_{1}(1+\alpha \mathrm{u})\right) \exp \left(\mathrm{u} \sum_{\mathrm{i}=1}^{\mathrm{n}} \ln \mathrm{x}_{\mathrm{i}}\right.}{\Gamma(\mathrm{u}+1)(\Gamma(\mathrm{u}))^{\mathrm{n}} \mathrm{v}^{\mathrm{nu}}}\right)}{\sum_{\mathrm{u}=1}^{\infty}\left(\frac{(1+\alpha u)^{\mathrm{u}-1} \theta_{1}^{\mathrm{u}} \exp \left(-\theta_{1}(1+\alpha \mathrm{u})\right) \exp \left(\mathrm{u} \sum_{\mathrm{i}=1}^{\mathrm{n}} \ln \mathrm{x}_{\mathrm{i}}\right.}{\Gamma(\mathrm{u}+1)(\Gamma(\mathrm{u}))^{\mathrm{n}} \mathrm{v}^{\mathrm{nu}}}\right)}
\end{aligned}
$$

The posterior variance of the Bayes estimator $\hat{\mathrm{u}}_{1} / \mathrm{X}$ is given by:

$$
\operatorname{Var}\left(\hat{\mathrm{u}}_{1} / \mathrm{X}\right)=
$$$$
\left(\frac{\sum_{\mathrm{u}=1}^{\infty}\left(\frac{\mathrm{u}^{2}(1+\alpha \mathrm{u})^{\mathrm{u}-1} \theta_{1}^{\mathrm{u}} \exp \left(-\theta_{1}(1+\alpha \mathrm{u})\right) \exp \left(\mathrm{u} \sum_{\mathrm{i}=1}^{\mathrm{n}} \ln \mathrm{x}_{\mathrm{i}}\right.}{\Gamma(\mathrm{u}+1)(\Gamma(\mathrm{u}))^{\mathrm{n}} \mathrm{v}^{\mathrm{nu}}}\right)}{\sum_{\mathrm{u}=1}^{\infty}\left(\frac{(1+\alpha \mathrm{u})^{\mathrm{u}-1} \theta_{1}^{\mathrm{u}} \exp \left(-\theta_{1}(1+\alpha \mathrm{u})\right) \exp \left(\mathrm{u} \sum_{\mathrm{i}=1}^{\mathrm{n}} \ln \mathrm{x}_{\mathrm{i}}\right.}{\Gamma(\mathrm{u}+1)(\Gamma(\mathrm{u}))^{\mathrm{n}} \mathrm{v}^{\mathrm{nu}}}\right)}\right)
$$

$$
-\left(\frac{\sum_{\mathrm{u}=1}^{\infty}\left(\frac{\mathrm{u}(1+\alpha \mathrm{u})^{\mathrm{u}-1} \theta_{1}^{\mathrm{u}} \exp \left(-\theta_{1}(1+\alpha \mathrm{u})\right) \exp \left(\mathrm{u} \sum_{\mathrm{i}=1}^{\mathrm{n}} \ln \mathrm{x}_{\mathrm{i}}\right.}{\Gamma(\mathrm{u}+1)(\Gamma(\mathrm{u}))^{\mathrm{n}} \mathrm{v}^{\mathrm{nu}}}\right)}{\sum_{\mathrm{u}=1}^{\infty}\left(\frac{(1+\alpha \mathrm{u})^{\mathrm{u}-1} \theta_{1}^{\mathrm{u}} \exp \left(-\theta_{1}(1+\alpha \mathrm{u})\right) \exp \left(\mathrm{u} \sum_{\mathrm{i}=1}^{\mathrm{n}} \ln \mathrm{x}_{\mathrm{i}}\right.}{\Gamma(\mathrm{u}+1)(\Gamma(\mathrm{u}))^{\mathrm{n}} \mathrm{v}^{\mathrm{nu}}}\right)}\right)^{2}
$$

\section{Special Cases}

For $\alpha=0$, the generalized truncated Poisson distribution reduces to the truncated Poisson distribution; therefore, the Bayes estimator for scale parameter $\mathrm{u}$ is given by

$$
\hat{\mathrm{u}}_{1}^{*} / \mathrm{X}=\frac{\sum_{\mathrm{u}=1}^{\infty}\left(\frac{\mathrm{u} \theta_{1}^{\mathrm{u}} \exp \left(-\theta_{1}\right) \exp \left(\mathrm{u} \sum_{\mathrm{i}=1}^{\mathrm{n}} \ln \mathrm{x}_{\mathrm{i}}\right.}{\Gamma(\mathrm{u}+1)(\Gamma(\mathrm{u}))^{\mathrm{n}} \mathrm{v}^{\mathrm{nu}}}\right)}{\sum_{\mathrm{u}=1}^{\infty}\left(\frac{\theta_{1}^{\mathrm{u}} \exp \left(-\theta_{1}\right) \exp \left(\mathrm{u} \sum_{\mathrm{i}=1}^{\mathrm{n}} \ln \mathrm{x}_{\mathrm{i}}\right.}{\Gamma(\mathrm{u}+1)(\Gamma(\mathrm{u}))^{\mathrm{n}} \mathrm{v}^{\mathrm{nu}}}\right)},
$$


and the posterior variance of the Bayes estimator $\hat{\mathrm{u}}_{1}^{*} / \mathrm{X}$ is given by

$$
\begin{aligned}
& \operatorname{Var}\left(\hat{\mathrm{u}}_{1}^{*} / \mathrm{X}\right)= \\
& \left(\frac{\sum_{\mathrm{u}=1}^{\infty}\left(\frac{\mathrm{u}^{2} \theta_{1}^{\mathrm{u}} \exp \left(-\theta_{1}\right) \exp \left(\mathrm{u} \sum_{\mathrm{i}=1}^{\mathrm{n}} \ln \mathrm{x}_{\mathrm{i}}\right.}{\Gamma(\mathrm{u}+1)(\Gamma(\mathrm{u}))^{\mathrm{n}} \mathrm{v}^{\mathrm{nu}}}\right)}{\sum_{\mathrm{u}=1}^{\infty}\left(\frac{\theta_{1}^{\mathrm{u}} \exp \left(-\theta_{1}\right) \exp \left(\mathrm{u} \sum_{\mathrm{i}=1}^{\mathrm{n}} \ln \mathrm{x}_{\mathrm{i}}\right.}{\Gamma(\mathrm{u}+1)(\Gamma(\mathrm{u}))^{\mathrm{n}} \mathrm{v}^{\mathrm{nu}}}\right)}\right) \\
& -\left(\frac{\sum_{\mathrm{u}=1}^{\infty}\left(\frac{\mathrm{u} \theta_{1}^{\mathrm{u}} \exp \left(-\theta_{1}\right) \exp \left(\mathrm{u} \sum_{\mathrm{i}=1}^{\mathrm{n}} \ln \mathrm{x}_{\mathrm{i}}\right.}{\Gamma(\mathrm{u}+1)(\Gamma(\mathrm{u}))^{\mathrm{n}} \mathrm{v}^{\mathrm{nu}}}\right)}{\sum_{\mathrm{u}=1}^{\infty}\left(\frac{\theta_{1}^{\mathrm{u}} \exp \left(-\theta_{1}\right) \exp \left(\mathrm{u} \sum_{\mathrm{i}=1}^{\mathrm{n}} \ln \mathrm{x}_{\mathrm{i}}\right.}{\Gamma(\mathrm{u}+1)(\Gamma(\mathrm{u}))^{\mathrm{n}} \mathrm{v}^{\mathrm{nu}}}\right)}\right)^{2}
\end{aligned}
$$

Generalized Truncated Geometric Distribution as a Prior for Shape Parameter $\mathrm{u}$

If the prior assumed for the shape parameter is the Generalized Truncated Geometric distribution, then the pdf of the Generalized Truncated Geometric distribution is given by

$$
\begin{aligned}
& \mathrm{g}_{2}\left(\mathrm{u}, \theta_{2}\right)= \\
& \quad\left(1-\mathrm{m}+\theta_{2}\right)\left(\begin{array}{c}
\mathrm{mu}-1 \\
\mathrm{u}-1
\end{array}\right)\left(1-\theta_{2}\right)^{\mathrm{u}-1} \theta_{2}^{\mathrm{mu}-\mathrm{u}} ; \\
& \mathrm{u}=1,2,3, \ldots \ldots ; 0<\theta_{2}<1,
\end{aligned}
$$

and, for $\mathrm{m}=1$, the truncated geometric distribution with pdf results as

$$
\begin{aligned}
& \mathrm{g}_{2}^{*}\left(\mathrm{u}, \theta_{2}\right)=\theta_{2}\left(1-\theta_{2}\right)^{\mathrm{u}-1} \\
& \mathrm{u}=1,2,3, \ldots \ldots ; 0<\theta_{2}<1
\end{aligned}
$$

By combining likelihood function (3.1) and prior density function (5.1), the posterior distribution of $\mathrm{u}$, when the prior is the generalized truncated geometric distribution, is

$\mathrm{g}_{2}(\mathrm{u} / \mathrm{X})=$

$$
\begin{aligned}
& \underline{\left(\begin{array}{c}
m u-1 \\
\mathrm{u}-1
\end{array}\right)\left(1-\theta_{2}\right)^{\mathrm{u}-1} \theta_{2}^{\mathrm{mu}-\mathrm{u}} \exp \left(\mathrm{u} \sum_{\mathrm{i}=1}^{\mathrm{n}} \ln \mathrm{x}_{\mathrm{i}}\right)} \\
& (\Gamma(\mathrm{u}))^{\mathrm{n}} \mathrm{v}^{\mathrm{nu}} \\
& \sum_{\mathrm{u}=1}^{\infty}\left(\frac{\left(\begin{array}{c}
\mathrm{mu}-1 \\
\mathrm{u}-1
\end{array}\right)\left(1-\theta_{2}\right)^{\mathrm{u}-1} \theta_{2}^{\mathrm{mu}-\mathrm{u}} \exp \left(\mathrm{u} \sum_{\mathrm{i}=1}^{\mathrm{n}} \ln \mathrm{x}_{\mathrm{i}}\right)}{(\Gamma(\mathrm{u}))^{\mathrm{n}} \mathrm{v}^{\mathrm{nu}}}\right)
\end{aligned}
$$

$\mathrm{u}=1,2,3, \ldots$

Under a squared error loss function with prior $\mathrm{g}_{2}\left(\mathrm{u}, \theta_{2}\right)$, the Bayes estimator is

$\hat{\mathrm{u}}_{2} / \mathrm{X}=$

$\frac{\sum_{\mathrm{u}=1}^{\infty}\left(\frac{\mathrm{u}\left(\begin{array}{c}\mathrm{mu}-1 \\ \mathrm{u}-1\end{array}\right)\left(1-\theta_{2}\right)^{\mathrm{u}-1} \theta_{2}^{\mathrm{mu}-\mathrm{u}} \exp \left(\mathrm{u} \sum_{\mathrm{i}=1}^{\mathrm{n}} \ln \mathrm{x}_{\mathrm{i}}\right)}{(\Gamma(\mathrm{u}))^{\mathrm{n}} \mathrm{v}^{\mathrm{nu}}}\right)}{\sum_{\mathrm{u}=1}^{\infty}\left(\frac{\left(\begin{array}{c}\mathrm{mu}-1 \\ \mathrm{u}-1\end{array}\right)\left(1-\theta_{2}\right)^{\mathrm{u}-1} \theta_{2}^{\mathrm{mu}-\mathrm{u}} \exp \left(\mathrm{u} \sum_{\mathrm{i}=1}^{\mathrm{n}} \ln \mathrm{x}_{\mathrm{i}}\right)}{(\Gamma(\mathrm{u}))^{\mathrm{n}} \mathrm{v}^{\mathrm{nu}}}\right)}$,

and the posterior variance of the Bayes estimator $\hat{\mathrm{u}}_{2}^{*} / \mathrm{X}$ is given by 


$$
\begin{aligned}
& \operatorname{Var}\left(\hat{\mathrm{u}}_{2} / \mathrm{X}\right)= \\
& \left.\left(\frac{\sum_{u=1}^{\infty}\left(\frac{u^{2}\left(\begin{array}{c}
m u-1 \\
\mathrm{u}-1
\end{array}\right)\left(1-\theta_{2}\right)^{\mathrm{u}-1} \theta_{2}^{\mathrm{mu}-\mathrm{u}} \exp \left(\mathrm{u} \sum_{\mathrm{i}=1}^{\mathrm{n}} \ln \mathrm{x}_{\mathrm{i}}\right)}{(\Gamma(\mathrm{u}))^{\mathrm{n}} \mathrm{v}^{\mathrm{nu}}}\right)}{\sum_{\mathrm{u}=1}^{\infty}\left(\frac{\left(\begin{array}{c}
\mathrm{mu}-1 \\
\mathrm{u}-1
\end{array}\right)\left(1-\theta_{2}\right)^{\mathrm{u}-1} \theta_{2}^{\mathrm{mu}-\mathrm{u}} \exp \left(\mathrm{u} \sum_{\mathrm{i}=1}^{\mathrm{n}} \ln \mathrm{x}_{\mathrm{i}}\right)}{(\Gamma(\mathrm{u}))^{\mathrm{n}} \mathrm{v}^{\mathrm{nu}}}\right)}\right)\right) \\
& -\left(\frac{\sum_{\mathrm{u}=1}^{\infty}\left(\frac{\mathrm{u}\left(\begin{array}{c}
\mathrm{mu}-1 \\
\mathrm{u}-1
\end{array}\right)\left(1-\theta_{2}\right)^{\mathrm{u}-1} \theta_{2}^{\mathrm{mu}-\mathrm{u}} \exp \left(\mathrm{u} \sum_{\mathrm{i}=1}^{\mathrm{n}} \ln \mathrm{x}_{\mathrm{i}}\right)}{(\Gamma(\mathrm{u}))^{\mathrm{n}} \mathrm{v}^{\mathrm{nu}}}\right)}{\sum_{\mathrm{u}=1}^{\infty}\left(\frac{\left(\begin{array}{c}
\mathrm{mu}-1 \\
\mathrm{u}-1
\end{array}\right)\left(1-\theta_{2}\right)^{\mathrm{u}-1} \theta_{2}^{\mathrm{mu}-\mathrm{u}} \exp \left(\mathrm{u} \sum_{\mathrm{i}=1}^{\mathrm{n}} \ln \mathrm{x}_{\mathrm{i}}\right)}{(\Gamma(\mathrm{u}))^{\mathrm{n}} \mathrm{v}^{\mathrm{nu}}}\right)}\right)^{2} .
\end{aligned}
$$

Special Cases

For $\mathrm{m}=1$, the generalized truncated geometric distribution reduces to a truncated geometric distribution; therefore, the Bayes estimator for scale parameter $\mathrm{u}$ is given by:

$$
\hat{\mathrm{u}}_{2}^{*} / \mathrm{X}=\frac{\sum_{\mathrm{u}=1}^{\infty}\left(\frac{\mathrm{u}\left(1-\theta_{2}\right)^{\mathrm{u}-1} \exp \left(\mathrm{u} \sum_{\mathrm{i}=1}^{\mathrm{n}} \ln \mathrm{n}_{\mathrm{i}}\right)}{(\Gamma(\mathrm{u}))^{\mathrm{n}} \mathrm{v}^{\mathrm{nu}}}\right)}{\sum_{\mathrm{u}=1}^{\infty}\left(\frac{\left(1-\theta_{2}\right)^{\mathrm{u}-1} \exp \left(\mathrm{u} \sum_{\mathrm{i}=1}^{\mathrm{n}} \ln \mathrm{ln}_{\mathrm{i}}\right)}{(\Gamma(\mathrm{u}))^{\mathrm{n}} \mathrm{v}^{\mathrm{nu}}}\right)},
$$

and the posterior variance of Bayes estimator $\hat{\mathrm{u}}_{2}^{*} / \mathrm{X}$ is given by

$$
\begin{aligned}
& \operatorname{Var}\left(\hat{\mathrm{u}}_{2}^{*} / \mathrm{X}\right)= \\
& \left(\frac{\sum_{\mathrm{u}=1}^{\infty}\left(\frac{\mathrm{u}^{2}\left(1-\theta_{2}\right)^{\mathrm{u}-1} \exp \left(\mathrm{u} \sum_{\mathrm{i}=1}^{\mathrm{n}} \ln \mathrm{x}_{\mathrm{i}}\right)}{(\Gamma(\mathrm{u}))^{\mathrm{n}} \mathrm{v}^{\mathrm{nu}}}\right)}{\sum_{\mathrm{u}=1}^{\infty}\left(\frac{\left(1-\theta_{2}\right)^{\mathrm{u}-1} \exp \left(\mathrm{u} \sum_{\mathrm{i}=1}^{\mathrm{n}} \ln \mathrm{x}_{\mathrm{i}}\right)}{(\Gamma(\mathrm{u}))^{\mathrm{n}} \mathrm{v}^{\mathrm{nu}}}\right)}\right) \\
& -\left(\frac{\sum_{\mathrm{u}=1}^{\infty}\left(\frac{\mathrm{u}\left(1-\theta_{2}\right)^{\mathrm{u}-1} \exp \left(\mathrm{u} \sum_{\mathrm{i}=1}^{\mathrm{n}} \ln \mathrm{x}_{\mathrm{i}}\right)}{(\Gamma(\mathrm{u}))^{\mathrm{n}} \mathrm{v}^{\mathrm{nu}}}\right)}{\sum_{\mathrm{u}=1}^{\infty}\left(\frac{\left(1-\theta_{2}\right)^{\mathrm{u}-1} \exp \left(\mathrm{u} \sum_{\mathrm{i}=1}^{\mathrm{n}} \ln \mathrm{x}_{\mathrm{i}}\right)}{(\Gamma(\mathrm{u}))^{\mathrm{n}} \mathrm{v}^{\mathrm{nu}}}\right)}\right)^{2} .
\end{aligned}
$$

When both the shape and scale parameters are unknown, the different independent prior distributions are assumed for two unknown parameters $\mathrm{u}$ (shape) and $\mathrm{v}$ (scale) of Erlang distributions.

Posterior Distribution under Generalized Truncated Poisson and Inverted Gamma Priors

The assumed prior for the shape parameter $\mathrm{u}$ of the Erlang distribution is Generalized Truncated Poisson distribution having the pdf

$$
\begin{aligned}
& \mathrm{g}_{11}\left(\mathrm{u}, \theta_{1}\right)=\frac{(1+\alpha \mathrm{u})^{\mathrm{u}-1} \theta_{1}^{\mathrm{u}} \exp \left(-\theta_{1}(1+\alpha \mathrm{u})\right)}{\Gamma(\mathrm{u}+1)\left(1-\exp \left(-\theta_{1}\right)\right)} ; \\
& \mathrm{u}=1,2,3, \ldots ; \theta_{1}>0 .
\end{aligned}
$$

For the scale parameter $\mathrm{v}$, the assumed prior is the inverted Gamma distribution with pdf

$$
\begin{aligned}
& \mathrm{g}_{12}\left(\mathrm{v} ; \alpha_{1}, \beta_{1}\right)=\frac{\beta_{1}^{\alpha_{1}} \mathrm{v}^{-\left(\alpha_{1}+1\right)} \exp \left(-\mathrm{v}^{-1} \beta_{1}\right)}{\Gamma\left(\alpha_{1}\right)} \\
& \mathrm{v}>1, \alpha_{1}>0, \beta_{1}>0
\end{aligned}
$$


The joint prior distribution of $\mathrm{u}$ and $\mathrm{v}$ is defined as:

$$
\mathrm{g}_{1}(\mathrm{u}, \mathrm{v})=\mathrm{g}_{11}\left(\mathrm{u}, \theta_{1}\right) \cdot \mathrm{g}_{12}\left(\mathrm{v} ; \alpha_{1,} \beta_{1}\right) .
$$

By combining likelihood function (3.1) and joint prior function (6.3), the joint posterior distribution of $u$ and $v$ is given by (3.2.1.4). The marginal posterior distribution of $\mathrm{u}$ and $\mathrm{v}$, $\mathrm{g}_{1}\left(\mathrm{u}, \frac{\mathrm{v}}{\mathrm{X}}\right)$, are shown in (3.2.1.5) and (3.2.1.6). Under the squared loss function, the expression for Bayes estimators of $\mathrm{u}$ and $\mathrm{v}$ with their respective posterior variances are given by (3.2.1.7), (3.2.1.8), (3.2.1.9) and (3.2.1.10).

$$
\begin{aligned}
& (1+\alpha u)^{u-1} \theta_{1}^{u} \exp \left(-\theta_{1}(1+\alpha u)\right) \times \exp \left(u \sum_{i=1}^{n} \ln x_{i}\right) v^{-\left(a_{1}+n u+1\right)} \times \exp \left\{-v^{-1}\left(\beta_{1}+\sum_{i=1}^{n} x_{i}\right\}\right. \\
& \mathrm{g}_{1}\left(\mathrm{u}, \frac{\mathrm{v}}{\mathrm{X}}\right)=\frac{\Gamma(\mathrm{u}+1)(\Gamma(\mathrm{u}))^{\mathrm{n}}}{\sum_{\mathrm{u}=1}^{\infty}\left(\frac{(1+\alpha \mathrm{u})^{\mathrm{u}-1} \theta_{1}^{\mathrm{u}} \exp \left(-\theta_{1}(1+\alpha \mathrm{u})\right) \times \exp \left(\mathrm{u} \sum_{\mathrm{i}=1}^{\mathrm{n}} \ln \mathrm{x}_{\mathrm{i}}\right) \int_{\mathrm{o}}^{\infty} \mathrm{v}^{-\left(\alpha_{1}+n u+1\right)} \times \exp \left\{-\mathrm{v}^{-1}\left(\beta_{1}+\sum_{\mathrm{i}=1}^{\mathrm{n}} \mathrm{x}_{\mathrm{i}}\right\} \mathrm{db}\right.}{\Gamma(\mathrm{u}+1)(\Gamma(\mathrm{u}))^{\mathrm{n}}}\right)} \\
& =\frac{\left(\frac{(1+\alpha \mathrm{u})^{\mathrm{u}-1} \theta_{1}^{\mathrm{u}} \exp \left(-\theta_{1}(1+\alpha \mathrm{u})\right) \times \exp \left(\mathrm{u} \sum_{\mathrm{i}=1}^{\mathrm{n}} \ln \mathrm{x}_{\mathrm{i}}\right) \mathrm{v}^{-\left(\alpha_{1}+\mathrm{nu}+1\right)} \times \exp \left\{-\mathrm{v}^{-1}\left(\beta_{1}+\sum_{\mathrm{i}=1}^{\mathrm{n}} \mathrm{x}_{\mathrm{i}}\right\}\right.}{\Gamma(\mathrm{u}+1)(\Gamma(\mathrm{u}))^{\mathrm{n}}}\right)}{\sum_{\mathrm{u}=1}^{\infty}\left(\frac{(1+\alpha \mathrm{u})^{\mathrm{u}-1} \theta_{1}^{\mathrm{u}} \exp \left(-\theta_{1}(1+\alpha \mathrm{u})\right) \times \exp \left(\mathrm{u} \sum_{\mathrm{i}=1}^{\mathrm{n}} \ln _{\mathrm{i}}\right) \Gamma\left(\alpha_{1}+\mathrm{nu}\right)}{\Gamma(\mathrm{u}+1)(\Gamma(\mathrm{u}))^{\mathrm{n}}\left(\beta_{1}+\sum_{\mathrm{i}-1}^{\mathrm{n}} \mathrm{x}_{\mathrm{i}}\right)^{\alpha_{1}+\mathrm{nu}}}\right)} \\
& \begin{aligned}
& \mathrm{g}_{1}\left(\frac{\mathrm{u}}{\mathrm{X}}\right)= \frac{(1+\alpha \mathrm{u})^{\mathrm{u}-1} \theta_{1}^{\mathrm{u}} \exp \left(-\theta_{1}(1+\alpha \mathrm{u})\right) \exp \left(\mathrm{u} \sum_{\mathrm{i}=1}^{\mathrm{n}} \ln \mathrm{x}_{\mathrm{i}}\right) \int_{\mathrm{o}}^{\infty} \mathrm{v}^{-\left(\alpha_{1}+\mathrm{nu}+1\right)} \exp \left\{-\mathrm{v}^{-1}\left(\beta_{1}+\sum_{\mathrm{i}=1}^{\mathrm{n}} \mathrm{x}_{\mathrm{i}}\right\} \mathrm{db}\right.}{\Gamma(\mathrm{u}+1)(\Gamma(\mathrm{u}))^{\mathrm{n}}} \\
& \sum_{\mathrm{u}=1}^{\infty}\left(\frac{(1+\alpha \mathrm{u})^{\mathrm{u}-1} \theta_{1}^{\mathrm{u}} \exp \left(-\theta_{1}(1+\alpha \mathrm{u})\right) \exp \left(\mathrm{u} \sum_{\mathrm{i}=1}^{\mathrm{n}} \ln \mathrm{x}_{\mathrm{i}}\right) \Gamma\left(\alpha_{1}+\mathrm{nu}\right)}{\Gamma(\mathrm{u}+1)(\Gamma(\mathrm{u}))^{\mathrm{n}}\left(\beta_{1}+\sum_{\mathrm{i}-1}^{\mathrm{n}} \mathrm{x}_{\mathrm{i}}\right)^{\alpha_{1}+\mathrm{nu}}}\right) \frac{(1+\alpha \mathrm{u})^{\mathrm{u}-1} \theta_{1}^{\mathrm{u}} \exp \left(-\theta_{1}(1+\alpha \mathrm{u})\right) \exp \left(\mathrm{u} \sum_{\mathrm{i}=1}^{\mathrm{n}} \ln \mathrm{x}_{\mathrm{i}}\right) \Gamma\left(\alpha_{1}+\mathrm{nu}\right)}{\Gamma(\mathrm{u}+1)(\Gamma(\mathrm{u}))^{\mathrm{n}}\left(\beta_{1}+\sum_{\mathrm{i}-1}^{\mathrm{n}} \mathrm{x}_{\mathrm{i}}\right)^{\alpha_{1}+n \mathrm{nu}}} \\
& \sum_{\mathrm{u}=1}^{\infty}\left(\frac{(1+\alpha \mathrm{u})^{\mathrm{u}-1} \theta_{1}^{\mathrm{u}} \exp \left(-\theta_{1}(1+\alpha \mathrm{u})\right) \exp \left(\mathrm{u} \sum_{\mathrm{i}=1}^{\mathrm{n}} \ln \mathrm{x}_{\mathrm{i}}\right) \Gamma\left(\alpha_{1}+\mathrm{nu}\right)}{\Gamma(\mathrm{u}+1)(\Gamma(\mathrm{u}))^{\mathrm{n}}\left(\beta_{1}+\sum_{\mathrm{i}-1}^{\mathrm{n}} \mathrm{x}_{\mathrm{i}}\right)^{\alpha_{1}+\mathrm{nu}}}\right)
\end{aligned} \\
& \mathrm{u}=1,2, \ldots
\end{aligned}
$$




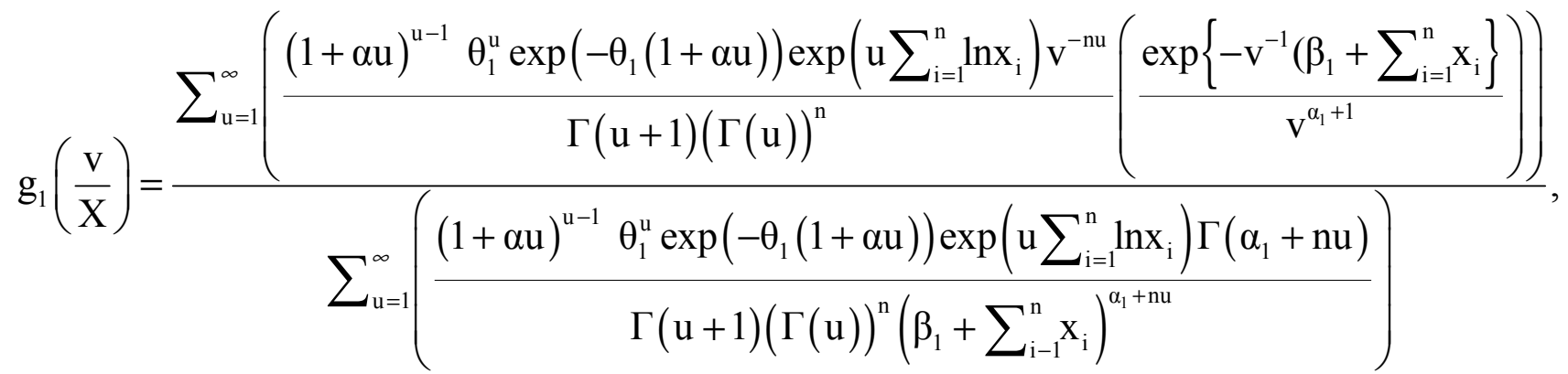

$\mathrm{v}>0$

$$
\hat{\mathrm{u}}_{1} / \mathrm{X}=\frac{\sum_{\mathrm{u}=1}^{\infty}\left(\frac{\mathrm{u}(1+\alpha \mathrm{u})^{\mathrm{u}-1} \theta_{1}^{\mathrm{u}} \exp \left(-\theta_{1}(1+\alpha \mathrm{u})\right) \exp \left(\mathrm{u} \sum_{\mathrm{i}=1}^{\mathrm{n}} \ln \mathrm{x}_{\mathrm{i}}\right) \Gamma\left(\alpha_{1}+n \mathrm{u}\right)}{\Gamma(\mathrm{u}+1)(\Gamma(\mathrm{u}))^{\mathrm{n}}\left(\beta_{1}+\sum_{\mathrm{i}-1}^{\mathrm{n}} \mathrm{x}_{\mathrm{i}}\right)^{\alpha_{1}+\mathrm{nu}}}\right)}{\sum_{\mathrm{u}=1}^{\infty}\left(\frac{(1+\alpha \mathrm{u})^{\mathrm{u}-1} \theta_{1}^{\mathrm{u}} \exp \left(-\theta_{1}(1+\alpha \mathrm{u})\right) \exp \left(\mathrm{u} \sum_{\mathrm{i}=1}^{\mathrm{n}} \ln \mathrm{x}_{\mathrm{i}}\right) \Gamma\left(\alpha_{1}+\mathrm{nu}\right)}{\Gamma(\mathrm{u}+1)(\Gamma(\mathrm{u}))^{\mathrm{n}}\left(\beta_{1}+\sum_{\mathrm{i}-1}^{\mathrm{n}} \mathrm{x}_{\mathrm{i}}\right)^{\alpha_{1}+\mathrm{nu}}}\right)}
$$

$$
\begin{gathered}
\hat{\mathrm{v}}_{1} / \mathrm{X}=\frac{\sum_{\mathrm{u}=1}^{\infty}\left(\frac{(1+\alpha \mathrm{u})^{\mathrm{u}-1} \theta_{1}^{\mathrm{u}} \exp \left(-\theta_{1}(1+\alpha \mathrm{u})\right) \exp \left(\mathrm{u} \sum_{\mathrm{i}=1}^{\mathrm{n}} \ln \mathrm{x}_{\mathrm{i}}\right)}{\Gamma(\mathrm{u}+1)(\Gamma(\mathrm{u}))^{\mathrm{n}}}\right)\left(\int_{0}^{\infty} \frac{\operatorname{vexp}\left\{-\mathrm{v}^{-1}\left(\beta_{1}+\sum_{\mathrm{i}=1}^{\mathrm{n}} \mathrm{x}_{\mathrm{i}}\right\}\right.}{\mathrm{v}^{\alpha_{1}+\mathrm{nu}+1}}\right)}{\sum_{\mathrm{u}=1}^{\infty}\left(\frac{(1+\alpha \mathrm{u})^{\mathrm{u}-1} \theta_{1}^{\mathrm{u}} \exp \left(-\theta_{1}(1+\alpha \mathrm{u})\right) \exp \left(\mathrm{u} \sum_{\mathrm{i}=1}^{\mathrm{n}} \ln \mathrm{x}_{\mathrm{i}}\right) \Gamma\left(\alpha_{1}+\mathrm{nu}\right)}{\Gamma(\mathrm{u}+1)(\Gamma(\mathrm{u}))^{\mathrm{n}}\left(\beta_{1}+\sum_{\mathrm{i}-1}^{\mathrm{n}} \mathrm{x}_{\mathrm{i}}\right)^{\alpha_{1}+\mathrm{nu}}}\right)} \\
\left.=\frac{(1+\alpha \mathrm{u})^{\mathrm{u}-1} \theta_{1}^{\mathrm{u}} \exp \left(-\theta_{1}(1+\alpha \mathrm{u})\right) \exp \left(\mathrm{u} \sum_{\mathrm{i}=1}^{\mathrm{n}} \ln \mathrm{x}_{\mathrm{i}}\right) \Gamma\left(\alpha_{1}+\mathrm{nu}-1\right)}{\Gamma(\mathrm{u}+1)(\Gamma(\mathrm{u}))^{\mathrm{n}}\left(\beta_{1}+\sum_{\mathrm{i}-1}^{\mathrm{n}} \mathrm{x}_{\mathrm{i}}\right)^{\alpha_{1}+\mathrm{nu}-1}}\right) \\
\sum_{\mathrm{u}=1}^{\infty}\left(\frac{(1+\alpha \mathrm{u})^{\mathrm{u}-1} \theta_{1}^{\mathrm{u}} \exp \left(-\theta_{1}(1+\alpha \mathrm{u})\right) \exp \left(\mathrm{u} \sum_{\mathrm{i}=1}^{\mathrm{n}} \ln \mathrm{x}_{\mathrm{i}}\right) \Gamma\left(\alpha_{1}+\mathrm{nu}\right)}{\Gamma(\mathrm{u}+1)(\Gamma(\mathrm{u}))^{\mathrm{n}}\left(\beta_{1}+\sum_{\mathrm{i}-1}^{\mathrm{n}} \mathrm{x}_{\mathrm{i}}\right)^{\alpha_{1}+\mathrm{nu}}}\right)
\end{gathered}
$$




$$
\begin{aligned}
& \operatorname{Var}\left(\hat{\mathrm{u}}_{1} / \mathrm{X}\right)=\left(\frac{\sum_{\mathrm{u}=1}^{\infty}\left(\frac{\mathrm{u}^{2}(1+\alpha \mathrm{u})^{\mathrm{u}-1} \theta_{1}^{\mathrm{u}} \exp \left(-\theta_{1}(1+\alpha \mathrm{u})\right) \exp \left(\mathrm{u} \sum_{\mathrm{i}=1}^{\mathrm{n}} \ln \mathrm{x}_{\mathrm{i}}\right) \Gamma\left(\alpha_{1}+\mathrm{nu}\right)}{\Gamma(\mathrm{u}+1)(\Gamma(\mathrm{u}))^{\mathrm{n}}\left(\beta_{1}+\sum_{\mathrm{i}-1}^{\mathrm{n}} \mathrm{x}_{\mathrm{i}}\right)^{\alpha_{1}+\mathrm{nu}}}\right)}{\sum_{\mathrm{u}=1}^{\infty}\left(\frac{(1+\alpha \mathrm{u})^{\mathrm{u}-1} \theta_{1}^{\mathrm{u}} \exp \left(-\theta_{1}(1+\alpha \mathrm{u})\right) \exp \left(\mathrm{u} \sum_{\mathrm{i}=1}^{\mathrm{n}} \ln \mathrm{x}_{\mathrm{i}}\right) \Gamma\left(\alpha_{1}+\mathrm{nu}\right)}{\Gamma(\mathrm{u}+1)(\Gamma(\mathrm{u}))^{\mathrm{n}}\left(\beta_{1}+\sum_{\mathrm{i}-1}^{\mathrm{n}} \mathrm{x}_{\mathrm{i}}\right)^{\alpha_{1}+\mathrm{nu}}}\right)}\right) \\
& -\left(\frac{\sum_{\mathrm{u}=1}^{\infty}\left(\frac{\mathrm{u}(1+\alpha \mathrm{u})^{\mathrm{u}-1} \theta_{1}^{\mathrm{u}} \exp \left(-\theta_{1}(1+\alpha \mathrm{u})\right) \exp \left(\mathrm{u} \sum_{\mathrm{i}=1}^{\mathrm{n}} \ln \mathrm{x}_{\mathrm{i}}\right) \Gamma\left(\alpha_{1}+\mathrm{nu}\right)}{\Gamma(\mathrm{u}+1)(\Gamma(\mathrm{u}))^{\mathrm{n}}\left(\beta_{1}+\sum_{\mathrm{i}-1}^{\mathrm{n}} \mathrm{x}_{\mathrm{i}}\right)^{\alpha_{1}+\mathrm{nu}}}\right)}{\sum_{\mathrm{u}=1}^{\infty}\left(\frac{(1+\alpha \mathrm{u})^{\mathrm{u}-1} \theta_{1}^{\mathrm{u}} \exp \left(-\theta_{1}(1+\alpha u)\right) \exp \left(\mathrm{u} \sum_{\mathrm{i}=1}^{\mathrm{n}} \ln \mathrm{x}_{\mathrm{i}}\right) \Gamma\left(\alpha_{1}+\mathrm{nu}\right)}{\Gamma(\mathrm{u}+1)(\Gamma(\mathrm{u}))^{\mathrm{n}}\left(\beta_{1}+\sum_{\mathrm{i}-1}^{\mathrm{n}} \mathrm{x}_{\mathrm{i}}\right)^{\alpha_{1}+\mathrm{nu}}}\right)}\right)^{2}
\end{aligned}
$$

$$
\begin{aligned}
\operatorname{Var}\left(\hat{\mathrm{v}}_{1} / \mathrm{X}\right)= & \frac{\sum_{\mathrm{u}=1}^{\infty}\left(\frac{(1+\alpha \mathrm{u})^{\mathrm{u}-1} \theta_{1}^{\mathrm{u}} \exp \left(-\theta_{1}(1+\alpha \mathrm{u})\right) \exp \left(\mathrm{u} \sum_{\mathrm{i}=1}^{\mathrm{n}} \ln \mathrm{x}_{\mathrm{i}}\right)}{\Gamma(\mathrm{u}+1)(\Gamma(\mathrm{u}))^{\mathrm{n}}} \int_{0}^{\infty} \frac{\operatorname{vexp}\left\{-\mathrm{v}^{-1}\left(\beta_{1}+\sum_{\mathrm{i}=1}^{\mathrm{n}} \mathrm{x}_{\mathrm{i}}\right\}\right.}{\mathrm{v}^{\alpha_{1}+\mathrm{nu}+1}}\right)}{\sum_{\mathrm{u}=1}^{\infty}\left(\frac{(1+\alpha \mathrm{u})^{\mathrm{u}-1} \theta_{1}^{\mathrm{u}} \exp \left(-\theta_{1}(1+\alpha \mathrm{u})\right) \exp \left(\mathrm{u} \sum_{\mathrm{i}=1}^{\mathrm{n}} \operatorname{lnx}_{\mathrm{i}}\right) \Gamma\left(\alpha_{1}+\mathrm{nu}\right)}{\Gamma(\mathrm{u}+1)(\Gamma(\mathrm{u}))^{\mathrm{n}}\left(\beta_{1}+\sum_{\mathrm{i}-1}^{\mathrm{n}} \mathrm{x}_{\mathrm{i}}\right)^{\alpha_{1}+\mathrm{nu}}}\right)} \\
& -\left(\frac{\frac{(1+\alpha \mathrm{u})^{\mathrm{u}-1} \theta_{1}^{\mathrm{u}} \exp \left(-\theta_{1}(1+\alpha \mathrm{u})\right) \exp \left(\mathrm{u} \sum_{\mathrm{i}=1}^{\mathrm{n}} \ln \mathrm{x}_{\mathrm{i}}\right) \Gamma\left(\alpha_{1}+\mathrm{nu}-1\right)}{\Gamma(\mathrm{u}+1)(\Gamma(\mathrm{u}))^{\mathrm{n}}\left(\beta_{1}+\sum_{\mathrm{i}-1}^{\mathrm{n}} \mathrm{x}_{\mathrm{i}}\right)^{\alpha_{1}+\mathrm{nu}-1}}}{\sum_{\mathrm{u}=1}^{\infty}\left(\frac{(1+\alpha \mathrm{u})^{\mathrm{u}-1} \theta_{1}^{\mathrm{u}} \exp \left(-\theta_{1}(1+\alpha \mathrm{u})\right) \exp \left(\mathrm{u} \sum_{\mathrm{i}=1}^{\mathrm{n}} \ln \mathrm{x}_{\mathrm{i}}\right) \Gamma\left(\alpha_{1}+\mathrm{nu}\right)}{\Gamma(\mathrm{u}+1)(\Gamma(\mathrm{u}))^{\mathrm{n}}\left(\beta_{1}+\sum_{\mathrm{i}-1}^{\mathrm{n}} \mathrm{x}_{\mathrm{i}}\right)^{\alpha_{1}+\mathrm{nu}}}\right)}\right)^{2}
\end{aligned}
$$




$$
\begin{aligned}
\operatorname{Var}\left(\hat{\mathrm{v}}_{1} / \mathrm{X}\right)= & \frac{\sum_{\mathrm{u}=1}^{\infty}\left(\frac{(1+\alpha \mathrm{u})^{\mathrm{u}-1} \theta_{1}^{\mathrm{u}} \exp \left(-\theta_{1}(1+\alpha \mathrm{u})\right) \exp \left(\mathrm{u} \sum_{\mathrm{i}=1}^{\mathrm{n}} \ln \mathrm{x}_{\mathrm{i}}\right) \Gamma\left(\alpha_{1}+\mathrm{nu}-2\right)}{\Gamma(\mathrm{u}+1)(\Gamma(\mathrm{u}))^{\mathrm{n}}\left(\beta_{1}+\sum_{\mathrm{i}-1}^{\mathrm{n}} \mathrm{x}_{\mathrm{i}}\right)^{\alpha_{1}+\mathrm{nu}-2}}\right)}{\sum_{\mathrm{u}=1}^{\infty}\left(\frac{(1+\alpha \mathrm{u})^{\mathrm{u}-1} \theta_{1}^{\mathrm{u}} \exp \left(-\theta_{1}(1+\alpha \mathrm{u})\right) \exp \left(\mathrm{u} \sum_{\mathrm{i}=1}^{\mathrm{n}} \ln \mathrm{x}_{\mathrm{i}}\right) \Gamma\left(\alpha_{1}+\mathrm{nu}\right)}{\Gamma(\mathrm{u}+1)(\Gamma(\mathrm{u}))^{\mathrm{n}}\left(\beta_{1}+\sum_{\mathrm{i}-1}^{\mathrm{n}} \mathrm{x}_{\mathrm{i}}\right)^{\alpha_{1}+\mathrm{nu}}}\right)} \\
& -\left(\frac{\frac{(1+\alpha \mathrm{u})^{\mathrm{u}-1} \theta_{1}^{\mathrm{u}} \exp \left(-\theta_{1}(1+\alpha \mathrm{u})\right) \exp \left(\mathrm{u} \sum_{\mathrm{i}=1}^{\mathrm{n}} \operatorname{lnx}_{\mathrm{i}}\right) \Gamma\left(\alpha_{1}+\mathrm{nu}-1\right)}{\Gamma(\mathrm{u}+1)(\Gamma(\mathrm{u}))^{\mathrm{n}}\left(\beta_{1}+\sum_{\mathrm{i}-1}^{\mathrm{n}} \mathrm{x}_{\mathrm{i}}\right)^{\alpha_{1}+\mathrm{nu}-1}}}{\sum_{\mathrm{u}=1}^{\infty}\left(\frac{(1+\alpha \mathrm{u})^{\mathrm{u}-1} \theta_{1}^{\mathrm{u}} \exp \left(-\theta_{1}(1+\alpha \mathrm{u})\right) \exp \left(\mathrm{u} \sum_{\mathrm{i}=1}^{\mathrm{n}} \ln _{\mathrm{i}}\right) \Gamma\left(\alpha_{1}+\mathrm{nu}\right)}{\Gamma(\mathrm{u}+1)(\Gamma(\mathrm{u}))^{\mathrm{n}}\left(\beta_{1}+\sum_{\mathrm{i}-1}^{\mathrm{n}} \mathrm{x}_{\mathrm{i}}\right)^{\alpha_{1}+\mathrm{nu}}}\right)}\right)^{2}
\end{aligned}
$$

\section{Special Cases}

For $\alpha=0$, the generalized truncated Poisson distribution reduces to the truncated Poisson distribution, therefore, the Bayes estimator for scale parameter $u$ and shape parameter $\mathrm{v}$ are given by

$$
\begin{aligned}
& \hat{\mathrm{u}}_{1}^{*} / \mathrm{X}= \\
& \frac{\sum_{\mathrm{u}=1}^{\infty}\left(\frac{\mathrm{u} \theta_{1}^{\mathrm{u}} \exp \left(-\theta_{1}\right) \exp \left(\mathrm{u} \sum_{\mathrm{i}=1}^{\mathrm{n}} \ln \mathrm{x}_{\mathrm{i}}\right) \Gamma\left(\alpha_{1}+\mathrm{nu}\right)}{\Gamma(\mathrm{u}+1)(\Gamma(\mathrm{u}))^{\mathrm{n}}\left(\beta_{1}+\sum_{\mathrm{i}-1}^{\mathrm{n}} \mathrm{x}_{\mathrm{i}}\right)^{\alpha_{1}+\mathrm{nu}}}\right)}{\sum_{\mathrm{u}=1}^{\infty}\left(\frac{\theta_{1}^{\mathrm{u}} \exp \left(-\theta_{1}\right) \exp \left(\mathrm{u} \sum_{\mathrm{i}=1}^{\mathrm{n}} \ln \mathrm{x}_{\mathrm{i}}\right) \Gamma\left(\alpha_{1}+\mathrm{nu}\right)}{\Gamma(\mathrm{u}+1)(\Gamma(\mathrm{u}))^{\mathrm{n}}\left(\beta_{1}+\sum_{\mathrm{i}-1}^{\mathrm{n}} \mathrm{x}_{\mathrm{i}}\right)^{\alpha_{1}+\mathrm{nu}}}\right)}
\end{aligned}
$$

and

$$
\hat{\mathrm{v}}_{1}^{*} / \mathrm{X}=\frac{\frac{\theta_{1}^{\mathrm{u}} \exp \left(\mathrm{u} \sum_{\mathrm{i}=1}^{\mathrm{n}} \ln \mathrm{x}_{\mathrm{i}}\right) \Gamma\left(\alpha_{1}+\mathrm{nu}-1\right)}{\Gamma(\mathrm{u}+1)(\Gamma(\mathrm{u}))^{\mathrm{n}}\left(\beta_{1}+\sum_{\mathrm{i}-1}^{\mathrm{n}} \mathrm{x}_{\mathrm{i}}\right)^{\alpha_{1}+\mathrm{nu}-1}}}{\sum_{\mathrm{u}=1}^{\infty}\left(\frac{\theta_{1}^{\mathrm{u}} \exp \left(\mathrm{u} \sum_{\mathrm{i}=1}^{\mathrm{n}} \ln \mathrm{x}_{\mathrm{i}}\right) \Gamma\left(\alpha_{1}+\mathrm{nu}\right)}{\Gamma(\mathrm{u}+1)(\Gamma(\mathrm{u}))^{\mathrm{n}}\left(\beta_{1}+\sum_{\mathrm{i}-1}^{\mathrm{n}} \mathrm{x}_{\mathrm{i}}\right)^{\alpha_{1}+\mathrm{nu}}}\right)}
$$

In addition, the respective posterior variances of $\mathrm{u}$ and $\mathrm{v}$ are 


$$
\begin{aligned}
& \operatorname{Var}\left(\hat{\mathrm{u}}_{1}^{*} / \mathrm{X}\right)= \\
& \left(\frac{\sum_{\mathrm{u}=1}^{\infty}\left(\frac{\mathrm{u}^{2} \theta_{1}^{\mathrm{u}} \exp \left(\mathrm{u} \sum_{\mathrm{i}=1}^{\mathrm{n}} \ln \mathrm{x}_{\mathrm{i}}\right) \Gamma\left(\alpha_{1}+\mathrm{nu}\right)}{\Gamma(\mathrm{u}+1)(\Gamma(\mathrm{u}))^{\mathrm{n}}\left(\beta_{1}+\sum_{\mathrm{i}-1}^{\mathrm{n}} \mathrm{x}_{\mathrm{i}}\right)^{\alpha_{1}+\mathrm{nu}}}\right)}{\sum_{\mathrm{u}=1}^{\infty}\left(\frac{\theta_{1}^{\mathrm{u}} \exp \left(\mathrm{u} \sum_{\mathrm{i}=1}^{\mathrm{n}} \ln \mathrm{x}_{\mathrm{i}}\right) \Gamma\left(\alpha_{1}+\mathrm{nu}\right)}{\Gamma(\mathrm{u}+1)(\Gamma(\mathrm{u}))^{\mathrm{n}}\left(\beta_{1}+\sum_{\mathrm{i}-1}^{\mathrm{n}} \mathrm{x}_{\mathrm{i}}\right)^{\alpha_{1}+\mathrm{nu}}}\right)}\right) \\
& -\left(\frac{\sum_{\mathrm{u}=1}^{\infty}\left(\frac{\mathrm{u} \theta_{1}^{\mathrm{u}} \exp \left(\mathrm{u} \sum_{\mathrm{i}=1}^{\mathrm{n}} \ln \mathrm{x}_{\mathrm{i}}\right) \Gamma\left(\alpha_{1}+\mathrm{nu}\right)}{\Gamma(\mathrm{u}+1)(\Gamma(\mathrm{u}))^{\mathrm{n}}\left(\beta_{1}+\sum_{\mathrm{i}-1}^{\mathrm{n}} \mathrm{x}_{\mathrm{i}}\right)^{\alpha_{1}+\mathrm{nu}}}\right)}{\sum_{\mathrm{u}=1}^{\infty}\left(\frac{\theta_{1}^{\mathrm{u}} \exp \left(\mathrm{u} \sum_{\mathrm{i}=1}^{\mathrm{n}} \ln \mathrm{x}_{\mathrm{i}}\right) \Gamma\left(\alpha_{1}+\mathrm{nu}\right)}{\Gamma(\mathrm{u}+1)(\Gamma(\mathrm{u}))^{\mathrm{n}}\left(\beta_{1}+\sum_{\mathrm{i}-1}^{\mathrm{n}} \mathrm{x}_{\mathrm{i}}\right)^{\alpha_{1}+\mathrm{nu}}}\right)}\right)^{2}
\end{aligned}
$$

and

$$
\begin{aligned}
& \operatorname{Var}\left(\hat{\mathrm{V}}_{1}^{*} / \mathrm{X}\right)= \\
& \frac{\sum_{\mathrm{u}=1}^{\infty}\left(\frac{\theta_{1}^{\mathrm{u}} \exp \left(\mathrm{u} \sum_{\mathrm{i}=1}^{\mathrm{n}} \ln \mathrm{x}_{\mathrm{i}}\right) \Gamma\left(\alpha_{1}+\mathrm{nu}-2\right)}{\Gamma(\mathrm{u}+1)(\Gamma(\mathrm{u}))^{\mathrm{n}}\left(\beta_{1}+\sum_{\mathrm{i}-1}^{\mathrm{n}} \mathrm{x}_{\mathrm{i}}\right)^{\alpha_{1}+\mathrm{nu}-2}}\right)}{\sum_{\mathrm{u}=1}^{\infty}\left(\frac{\theta_{1}^{\mathrm{u}} \exp \left(\mathrm{u} \sum_{\mathrm{i}=1}^{\mathrm{n}} \ln \mathrm{x}_{\mathrm{i}}\right) \Gamma\left(\alpha_{1}+\mathrm{nu}\right)}{\Gamma(\mathrm{u}+1)(\Gamma(\mathrm{u}))^{\mathrm{n}}\left(\beta_{1}+\sum_{\mathrm{i}-1}^{\mathrm{n}} \mathrm{x}_{\mathrm{i}}\right)^{\alpha_{1}+\mathrm{nu}}}\right)} \\
& -\left(\frac{\frac{\theta_{1}^{\mathrm{u}} \exp \left(\mathrm{u} \sum_{\mathrm{i}=1}^{\mathrm{n}} \ln \mathrm{x}_{\mathrm{i}}\right) \Gamma\left(\alpha_{1}+\mathrm{nu}-1\right)}{\Gamma(\mathrm{u}+1)(\Gamma(\mathrm{u}))^{\mathrm{n}}\left(\beta_{1}+\sum_{\mathrm{i}-1}^{\mathrm{n}} \mathrm{x}_{\mathrm{i}}\right)^{\alpha_{1}+\mathrm{nu}-1}}}{\sum_{\mathrm{u}=1}^{\infty}\left(\frac{\theta_{1}^{\mathrm{u}} \exp \left(\mathrm{u} \sum_{\mathrm{i}=1}^{\mathrm{n}} \ln \mathrm{x}_{\mathrm{i}}\right) \Gamma\left(\alpha_{1}+\mathrm{nu}\right)}{\Gamma(\mathrm{u}+1)(\Gamma(\mathrm{u}))^{\mathrm{n}}\left(\beta_{1}+\sum_{\mathrm{i}-1}^{\mathrm{n}} \mathrm{x}_{\mathrm{i}}\right)^{\alpha_{1}+\mathrm{nu}}}\right)}\right)^{2}
\end{aligned}
$$

Posterior Distribution under Generalized Truncated Poisson and Gamma Priors

If the prior for $\mathrm{u}$ is assumed to be a generalized truncated Poisson distribution and the assumed prior for $\mathrm{v}$ is a Gamma distribution with pdfs:

$$
\begin{aligned}
& \mathrm{g}_{11}\left(\mathrm{u}_{1}, \theta_{1}\right)=\frac{(1+\alpha \mathrm{u})^{\mathrm{u}-1} \theta_{1}^{\mathrm{u}} \exp \left(-\theta_{1}(1+\alpha \mathrm{u})\right)}{\Gamma(\mathrm{u}+1)\left(1-\exp \left(-\theta_{1}\right)\right)} ; \\
& \mathrm{u}=1,2,3, \ldots ; \theta_{1}>0,
\end{aligned}
$$

and

$$
\begin{aligned}
& \mathrm{g}_{21}\left(\mathrm{v} ; \alpha_{2}, \beta_{2}\right)=\frac{\beta_{2}^{\alpha_{2}} \mathrm{v}^{-\left(\alpha_{2}+1\right)} \exp \left(-\mathrm{v}^{-1} \beta_{2}\right)}{\Gamma\left(\alpha_{2}\right)} \\
& \mathrm{v}>1, \alpha_{2}>0, \beta_{2}>0,
\end{aligned}
$$

then the joint Prior Distribution of $\mathrm{u}$ and $\mathrm{v}$ is defined as:

$$
\mathrm{g}_{2}(\mathrm{u}, \mathrm{v})=\mathrm{g}_{11}\left(\mathrm{u}_{1}, \theta_{1}\right) \cdot \mathrm{g}_{21}\left(\mathrm{v} ; \alpha_{2}, \beta_{2}\right)
$$

Combining likelihood function (3.1) with joint density (7.3), the joint posterior distributions of $\mathrm{u}$ and $\mathrm{v}$ are given by (3.2.2.4). The marginal posterior distribution of $\mathrm{u}$ and $\mathrm{v}$ are given by (3.2.2.5) and (3.2.2.6). The expression for Bayes estimators of $\mathrm{u}$ and $\mathrm{v}$ with their respective posterior variance are given in (3.2.2.7), (3.2.2.8), (3.2.2.9) and (3.2.2.10). 


$$
\begin{aligned}
& 2(1+\alpha u)^{u-1} \theta_{1}^{u} \exp \left(-\theta_{1}(1+\alpha u)\right) \int_{0}^{\infty} v^{\left(\alpha_{2}-n u\right)-1} \exp \left\{\frac{-1}{2}\left(\left(2 \beta_{2}\right) v+\frac{\left(2 \sum_{i=1}^{n} x_{i}\right)}{v}\right)\right\} d b \\
& \mathrm{~g}_{2}(\mathrm{u} / \mathrm{X})=\frac{\exp \left(-\mathrm{u} \sum_{\mathrm{i}=1}^{\mathrm{n}} \ln \mathrm{x}_{\mathrm{i}}\right) \Gamma(\mathrm{u}+1)(\Gamma(\mathrm{u}))^{\mathrm{n}}}{\sum_{\mathrm{u}=1}^{\infty}\left(\frac{2(1+\alpha \mathrm{u})^{\mathrm{u}-1} \theta_{1}^{\mathrm{u}} \exp \left(-\theta_{1}(1+\alpha \mathrm{u})\right) \exp \left(-\mathrm{u} \sum_{\mathrm{i}=1}^{\mathrm{n}} \ln _{\mathrm{i}}\right) \mathrm{K}_{2}\left(2 \sqrt{\beta_{2}\left(\sum \mathrm{x}_{\mathrm{i}}\right)}\right)}{\Gamma(\mathrm{u}+1)(\Gamma(\mathrm{u}))^{\mathrm{n}}\left(\sqrt{\left(\sum \mathrm{x}_{\mathrm{i}}\right)^{-1} \beta_{2}}\right)^{\alpha_{2}-\mathrm{nu}}}\right)} \\
& 2(1+\alpha u)^{\mathrm{u}-1} \theta_{1}^{\mathrm{u}} \exp \left(-\theta_{1}(1+\alpha \mathrm{u})\right) \exp \left(\mathrm{u} \sum_{\mathrm{i}=1}^{\mathrm{n}} \ln \mathrm{x}_{\mathrm{i}}\right) \mathrm{K}_{\alpha_{2-\mathrm{nu}}}\left(2 \sqrt{\beta_{2}\left(\sum \mathrm{x}_{\mathrm{i}}\right)}\right) \\
& \Gamma(\mathrm{u}+1)(\Gamma(\mathrm{u}))^{\mathrm{n}}\left(\left(\sum \mathrm{x}_{\mathrm{i}}\right)^{-1} \beta_{2}\right)^{\alpha_{2}-\mathrm{nu}} \\
& \mathrm{g}_{2}(\mathrm{u} / \mathrm{X})=\frac{2(1+\alpha \mathrm{u})^{\mathrm{u}-1} \theta_{1}^{\mathrm{u}} \exp \left(-\theta_{1}(1+\alpha \mathrm{u})\right) \exp \left(-\mathrm{u} \sum_{\mathrm{u}=1}^{\mathrm{n}} \ln _{\mathrm{i}}\right) \mathrm{K}_{\alpha-\mathrm{nu}}\left(2 \sqrt{\beta_{2}\left(\sum \mathrm{x}_{\mathrm{i}}\right)}\right)}{\Gamma(\mathrm{u}+1)(\Gamma(\mathrm{u}))^{\mathrm{n}}\left(\sqrt{\left(\sum \mathrm{x}_{\mathrm{i}}\right)^{-1} \beta_{2}}\right)^{\alpha_{2}-\mathrm{nu}}} ;
\end{aligned}
$$

$\mathrm{u}=1,2, .$.

$$
\mathrm{g}_{2}(\mathrm{v} / \mathrm{X})=\frac{\sum_{\mathrm{u}=1}^{\infty}\left(\frac{(1+\alpha \mathrm{u})^{\mathrm{u}-1} \theta_{1}^{\mathrm{u}} \exp \left(-\theta_{1}(1+\alpha \mathrm{u})\right) \mathrm{v}^{\left(\alpha_{2}-\mathrm{nu}\right)-1} \exp \left\{\frac{-1}{2}\left(\left(2 \beta_{2}\right) \mathrm{v}+\frac{\left(2 \sum_{\mathrm{i}=1}^{\mathrm{n}} \mathrm{x}_{\mathrm{i}}\right)}{\mathrm{v}}\right)\right\}}{\exp \left(-\mathrm{u} \sum_{\mathrm{i}=1}^{\mathrm{n}} \ln \mathrm{x}_{\mathrm{i}}\right) \Gamma(\mathrm{u}+1)(\Gamma(\mathrm{u}))^{\mathrm{n}}}\right)}{\sum_{\mathrm{u}=1}^{\infty}\left(\frac{2(1+\alpha \mathrm{u})^{\mathrm{u}-1} \theta_{1}^{\mathrm{u}} \exp \left(-\theta_{1}(1+\alpha \mathrm{u})\right) \exp \left(-\mathrm{u} \sum_{\mathrm{i}=1}^{\mathrm{n}} \ln \mathrm{x}_{\mathrm{i}}\right) \mathrm{K}_{\alpha_{2-\text { пu }}}\left(2 \sqrt{\beta_{2}\left(\sum \mathrm{x}_{\mathrm{i}}\right)}\right)}{\Gamma(\mathrm{u}+1)(\Gamma(\mathrm{u}))^{\mathrm{n}}\left(\sqrt{\left(\sum \mathrm{x}_{\mathrm{i}}\right)^{-1} \beta_{2}}\right)^{\alpha_{2}-\mathrm{nu}}} ;\right.}
$$

$\mathrm{v}>0$ 
ADIL H. KHAN \& T. R. JAN

$\hat{\mathrm{u}}_{2} / \mathrm{X}=\frac{\sum_{\mathrm{u}=1}^{\infty}\left(\frac{2 \mathrm{u}(1+\alpha \mathrm{u})^{\mathrm{u}-1} \theta_{1}^{\mathrm{u}} \exp \left(-\theta_{1}(1+\alpha \mathrm{u})\right) \exp \left(\mathrm{u} \sum_{\mathrm{i}=1}^{\mathrm{n}} \ln _{\mathrm{i}}\right) \mathrm{K}_{\alpha_{2-\mathrm{u}}}\left(2 \sqrt{\beta_{2}\left(\sum \mathrm{x}_{\mathrm{i}}\right)}\right)}{\Gamma(\mathrm{u}+1)(\Gamma(\mathrm{u}))^{\mathrm{n}}\left(\left(\sum \mathrm{x}_{\mathrm{i}}\right)^{-1} \beta_{2}\right)^{\alpha_{2}-\mathrm{nu}}}\right)}{\sum_{\mathrm{u}=1}^{\infty}\left(\frac{2(1+\alpha \mathrm{u})^{\mathrm{u}-1} \theta_{1}^{\mathrm{u}} \exp \left(-\theta_{1}(1+\alpha \mathrm{u})\right) \exp \left(-\mathrm{u} \sum_{\mathrm{i}=1}^{\mathrm{n}} \ln \mathrm{x}_{\mathrm{i}}\right) \mathrm{K}_{\alpha-\mathrm{nu}}\left(2 \sqrt{\beta_{2}\left(\sum \mathrm{x}_{\mathrm{i}}\right)}\right)}{\Gamma(\mathrm{u}+1)(\Gamma(\mathrm{u}))^{\mathrm{n}}\left(\sqrt{\left(\sum \mathrm{x}_{\mathrm{i}}\right)^{-1} \beta_{2}}\right)^{\alpha_{2}-\mathrm{nu}}}\right)}$

$\hat{\mathrm{v}}_{2} / \mathrm{X}=\frac{\sum_{\mathrm{u}=1}^{\infty}\left(\frac{(1+\alpha \mathrm{u})^{\mathrm{u}-1} \theta_{1}^{\mathrm{u}} \exp \left(-\theta_{1}(1+\alpha \mathrm{u})\right) \int_{0}^{\infty} \mathrm{v} \cdot \mathrm{v}^{\left(\alpha_{2}-\mathrm{nu}\right)-1} \exp \left\{\frac{-1}{2}\left(\left(2 \beta_{2}\right) \mathrm{v}+\frac{\left(2 \sum_{\mathrm{i}=1}^{\mathrm{n}} \mathrm{x}_{\mathrm{i}}\right)}{\mathrm{v}}\right)\right\} \mathrm{db}}{\exp \left(-\mathrm{u} \sum_{\mathrm{i}=1}^{\mathrm{n}} \ln \mathrm{x}_{\mathrm{i}}\right) \Gamma(\mathrm{u}+1)(\Gamma(\mathrm{u}))^{\mathrm{n}}}\right)}{\sum_{\mathrm{u}=1}^{\infty}\left(\frac{2(1+\alpha \mathrm{u})^{\mathrm{u}-1} \theta_{1}^{\mathrm{u}} \exp \left(-\theta_{1}(1+\alpha \mathrm{u})\right) \exp \left(-\mathrm{u} \sum_{\mathrm{i}=1}^{\mathrm{n}} \ln \mathrm{x}_{\mathrm{i}}\right) \mathrm{K}_{\alpha-\mathrm{nu}}\left(2 \sqrt{\beta_{2}\left(\sum \mathrm{x}_{\mathrm{i}}\right)}\right)}{\Gamma(\mathrm{u}+1)(\Gamma(\mathrm{u}))^{\mathrm{n}}\left(\sqrt{\left(\sum \mathrm{x}_{\mathrm{i}}\right)^{-1} \beta_{2}}\right)^{\alpha_{2}-\mathrm{nu}}}\right)}$

$\hat{\mathrm{v}}_{2} / \mathrm{X}=\frac{\sum_{\mathrm{u}=1}^{\infty}\left(\frac{2(1+\alpha \mathrm{u})^{\mathrm{u}-1} \theta_{1}^{\mathrm{u}} \exp \left(-\theta_{1}(1+\alpha \mathrm{u})\right) \exp \left(\mathrm{u} \sum_{\mathrm{i}=1}^{\mathrm{n}} \ln \mathrm{x}_{\mathrm{i}}\right) \mathrm{K}_{\mathrm{a}_{2-n+1}}\left(2 \sqrt{\beta_{2}\left(\sum \mathrm{x}_{\mathrm{i}}\right)}\right)}{\Gamma(\mathrm{u}+1)(\Gamma(\mathrm{u}))^{\mathrm{n}}\left(\sqrt{\left(\sum \mathrm{x}_{\mathrm{i}}\right)^{-1} \beta_{2}}\right)^{\alpha_{2}-\mathrm{nu}+1}}\right)}{\sum_{\mathrm{u}=1}^{\infty}\left(\frac{2(1+\alpha \mathrm{u})^{\mathrm{u}-1} \theta_{1}^{\mathrm{u}} \exp \left(-\theta_{1}(1+\alpha \mathrm{u})\right) \exp \left(-\mathrm{u} \sum_{\mathrm{i}=1}^{\mathrm{n}} \ln \mathrm{ln}_{\mathrm{i}}\right) \mathrm{K}_{\alpha-\mathrm{nu}}\left(2 \sqrt{\beta_{2}\left(\sum \mathrm{x}_{\mathrm{i}}\right)}\right)}{\Gamma(\mathrm{u}+1)(\Gamma(\mathrm{u}))^{\mathrm{n}}\left(\sqrt{\left(\sum \mathrm{x}_{\mathrm{i}}\right)^{-1} \beta_{2}}\right)^{\alpha_{2}-\mathrm{nu}}}\right)}$ 


$$
\begin{aligned}
& \operatorname{Var}\left(\hat{\mathrm{u}}_{2} / \mathrm{X}\right)=\frac{\sum_{\mathrm{u}=1}^{\infty}\left(\frac{2 \mathrm{u}^{2}(1+\alpha \mathrm{u})^{\mathrm{u}-1} \theta_{1}^{\mathrm{u}} \exp \left(-\theta_{1}(1+\alpha \mathrm{u})\right) \exp \left(\mathrm{u} \sum_{\mathrm{i}=1}^{\mathrm{n}} \ln \mathrm{x}_{\mathrm{i}}\right) \mathrm{K}_{\alpha_{2-\text {-nu }}}\left(2 \sqrt{\beta_{2}\left(\sum \mathrm{x}_{\mathrm{i}}\right)}\right)}{\Gamma(\mathrm{u}+1)(\Gamma(\mathrm{u}))^{\mathrm{n}}\left(\left(\sum \mathrm{x}_{\mathrm{i}}\right)^{-1} \beta_{2}\right)^{\alpha_{2}-\mathrm{nu}}}\right)}{\sum_{\mathrm{u}=1}^{\infty}\left(\frac{2(1+\alpha \mathrm{u})^{\mathrm{u}-1} \theta_{1}^{\mathrm{u}} \exp \left(-\theta_{1}(1+\alpha \mathrm{u})\right) \exp \left(-\mathrm{u} \sum_{\mathrm{i}=1}^{\mathrm{n}} \ln \mathrm{x}_{\mathrm{i}}\right) \mathrm{K}_{\alpha-\mathrm{nu}}\left(2 \sqrt{\beta_{2}\left(\sum \mathrm{x}_{\mathrm{i}}\right)}\right)}{\Gamma(\mathrm{u}+1)(\Gamma(\mathrm{u}))^{\mathrm{n}}\left(\sqrt{\left(\sum \mathrm{x}_{\mathrm{i}}\right)^{-1} \beta_{2}}\right)^{\alpha_{2}-\mathrm{nu}}}\right)} \\
& \left.-\left(\frac{\sum_{\mathrm{u}=1}^{\infty}\left(\frac{2 \mathrm{u}(1+\alpha \mathrm{u})^{\mathrm{u}-1} \theta_{1}^{\mathrm{u}} \exp \left(-\theta_{1}(1+\alpha \mathrm{u})\right) \exp \left(\mathrm{u} \sum_{\mathrm{i}=1}^{\mathrm{n}} \ln \mathrm{x}_{\mathrm{i}}\right) \mathrm{K}_{\alpha_{2-\text { - u }}}\left(2 \sqrt{\beta_{2}\left(\sum \mathrm{x}_{\mathrm{i}}\right)}\right)}{\Gamma(\mathrm{u}+1)(\Gamma(\mathrm{u}))^{\mathrm{n}}\left(\left(\sum \mathrm{x}_{\mathrm{i}}\right)^{-1} \beta_{2}\right)^{\alpha_{2}-\mathrm{nu}}}\right)}{\sum_{\mathrm{u}=1}^{\infty}\left(\frac{2(1+\alpha \mathrm{u})^{\mathrm{u}-1} \theta_{1}^{\mathrm{u}} \exp \left(-\theta_{1}(1+\alpha \mathrm{u})\right) \exp \left(-\mathrm{u} \sum_{\mathrm{i}=1}^{\mathrm{n}} \ln \mathrm{x}_{\mathrm{i}}\right) \mathrm{K}_{\alpha-\mathrm{nu}}\left(2 \sqrt{\beta_{2}\left(\sum \mathrm{x}_{\mathrm{i}}\right)}\right)}{\Gamma(\mathrm{u}+1)(\Gamma(\mathrm{u}))^{\mathrm{n}}\left(\sqrt{\left(\sum \mathrm{x}_{\mathrm{i}}\right)^{-1} \beta_{2}}\right)^{\alpha_{2}-\mathrm{nu}}}\right)}\right)\right)^{2}
\end{aligned}
$$

$$
\begin{aligned}
& \operatorname{Var}\left(\hat{\mathrm{v}}_{2} / \mathrm{X}\right)=\frac{\sum_{\mathrm{u}=1}^{\infty}\left(\frac{2(1+\alpha \mathrm{u})^{\mathrm{u}-1} \theta_{1}^{\mathrm{u}} \exp \left(-\theta_{1}(1+\alpha \mathrm{u})\right) \exp \left(\mathrm{u} \sum_{\mathrm{i}=1}^{\mathrm{n}} \ln \mathrm{x}_{\mathrm{i}}\right) \mathrm{K}_{\alpha_{2-\mathrm{n}+2}}\left(2 \sqrt{\beta_{2}\left(\sum \mathrm{x}_{\mathrm{i}}\right)}\right)}{\Gamma(\mathrm{u}+1)(\Gamma(\mathrm{u}))^{\mathrm{n}}\left(\sqrt{\left(\sum \mathrm{x}_{\mathrm{i}}\right)^{-1} \beta_{2}}\right)^{\alpha_{2}-\mathrm{nu}+2}}\right)}{\sum_{\mathrm{u}=1}^{\infty}\left(\frac{2(1+\alpha \mathrm{u})^{\mathrm{u}-1} \theta_{1}^{\mathrm{u}} \exp \left(-\theta_{1}(1+\alpha \mathrm{u})\right) \exp \left(-\mathrm{u} \sum_{\mathrm{i}=1}^{\mathrm{n}} \ln \mathrm{x}_{\mathrm{i}}\right) \mathrm{K}_{\alpha-\mathrm{nu}}\left(2 \sqrt{\beta_{2}\left(\sum \mathrm{x}_{\mathrm{i}}\right)}\right)}{\Gamma(\mathrm{u}+1)(\Gamma(\mathrm{u}))^{\mathrm{n}}\left(\sqrt{\left(\sum \mathrm{x}_{\mathrm{i}}\right)^{-1} \beta_{2}}\right)^{\alpha_{2}-\mathrm{nu}}}\right)} \\
& \left.-\left(\frac{\sum_{\mathrm{u}=1}^{\infty}\left(\frac{2(1+\alpha \mathrm{u})^{\mathrm{u}-1} \theta_{1}^{\mathrm{u}} \exp \left(-\theta_{1}(1+\alpha \mathrm{u})\right) \exp \left(\mathrm{u} \sum_{\mathrm{i}=1}^{\mathrm{n}} \ln \mathrm{x}_{\mathrm{i}}\right) \mathrm{K}_{\alpha_{2-\mathrm{nu}+1}}\left(2 \sqrt{\beta_{2}\left(\sum \mathrm{x}_{\mathrm{i}}\right)}\right)}{\Gamma(\mathrm{u}+1)(\Gamma(\mathrm{u}))^{\mathrm{n}}\left(\sqrt{\left(\sum \mathrm{x}_{\mathrm{i}}\right)^{-1} \beta_{2}}\right)^{\alpha_{2}-\mathrm{nu}+1}}\right)}{\sum_{\mathrm{u}=1}^{\infty}\left(\frac{2(1+\alpha \mathrm{u})^{\mathrm{u}-1} \theta_{1}^{\mathrm{u}} \exp \left(-\theta_{1}(1+\alpha \mathrm{u})\right) \exp \left(-\mathrm{u} \sum_{\mathrm{i}=1}^{\mathrm{n}} \ln \mathrm{x}_{\mathrm{i}}\right) \mathrm{K}_{\alpha-\mathrm{nu}}\left(2 \sqrt{\beta_{2}\left(\sum \mathrm{x}_{\mathrm{i}}\right)}\right)}{\Gamma(\mathrm{u}+1)(\Gamma(\mathrm{u}))^{\mathrm{n}}\left(\sqrt{\left(\sum \mathrm{x}_{\mathrm{i}}\right)^{-1} \beta_{2}}\right)^{\alpha_{2}-\mathrm{nu}}}\right)}\right)\right)^{2}
\end{aligned}
$$


Special Cases

For $\alpha=0$, the generalized truncated

Poisson distribution reduces to the truncated Poisson distribution, therefore, the Bayes estimator for scale parameter $\mathrm{u}$ and shape parameter $\mathrm{V}$ are given by (3.2.2.11) and (3.2.2.12). Their respective posterior variances are given by (3.2.2.13) and (3.2.2.14).

$$
\hat{\mathrm{u}}_{2}{ }^{*} / \mathrm{X}=\frac{\sum_{\mathrm{u}=1}^{\infty}\left(\frac{2 \mathrm{u} \theta_{1}^{\mathrm{u}} \exp \left(\mathrm{u} \sum_{\mathrm{i}=1}^{\mathrm{n}} \ln \mathrm{x}_{\mathrm{i}}\right) \mathrm{K}_{\mathrm{a}_{2-\mathrm{u}}}\left(2 \sqrt{\beta_{2}\left(\sum \mathrm{x}_{\mathrm{i}}\right)}\right)}{\Gamma(\mathrm{u}+1)(\Gamma(\mathrm{u}))^{\mathrm{n}}\left(\sqrt{\left(\sum \mathrm{x}_{\mathrm{i}}\right)^{-1} \beta_{2}}\right)^{a_{2}-\mathrm{nu}}}\right)}{\sum_{\mathrm{u}=1}^{\infty}\left(\frac{2 \theta_{1}^{\mathrm{u}} \exp \left(\mathrm{u} \sum_{\mathrm{i}=1}^{\mathrm{n}} \operatorname{lnx}_{\mathrm{i}}\right) \mathrm{K}_{\mathrm{a}_{2-\mathrm{u}}}\left(2 \sqrt{\beta_{2}\left(\sum \mathrm{x}_{\mathrm{i}}\right)}\right)}{\Gamma(\mathrm{u}+1)(\Gamma(\mathrm{u}))^{\mathrm{n}}\left(\sqrt{\left(\sum \mathrm{x}_{\mathrm{i}}\right)^{-1} \beta_{2}}\right)^{a_{2}-\mathrm{nu}}}\right)}
$$

$$
\hat{\mathrm{v}}_{2}^{*} / \mathrm{X}=\frac{\sum_{\mathrm{u}=1}^{\infty}\left(\frac{2 \theta_{1}^{\mathrm{u}} \exp \left(\mathrm{u} \sum_{\mathrm{i}=1}^{\mathrm{n}} \ln \mathrm{x}_{\mathrm{i}}\right) \mathrm{K}_{a_{2-m+1}}\left(2 \sqrt{\beta_{2}\left(\sum \mathrm{x}_{\mathrm{i}}\right)}\right)}{\Gamma(\mathrm{u}+1)(\Gamma(\mathrm{u}))^{\mathrm{n}}\left(\sqrt{\left(\sum \mathrm{x}_{\mathrm{i}}\right)^{-1} \beta_{2}}\right)^{\alpha_{2}-\mathrm{uu}+1}}\right)}{\sum_{\mathrm{u}=1}^{\infty}\left(\frac{2 \theta_{1}^{\mathrm{u}} \exp \left(-\mathrm{u} \sum_{\mathrm{i}=1}^{\mathrm{n}} \ln \mathrm{x}_{\mathrm{i}}\right) \mathrm{K}_{\mathrm{u}-\mathrm{nu}}\left(2 \sqrt{\beta_{2}\left(\sum \mathrm{x}_{\mathrm{i}}\right)}\right)}{\Gamma(\mathrm{u}+1)(\Gamma(\mathrm{u}))^{\mathrm{n}}\left(\sqrt{\left(\sum \mathrm{x}_{\mathrm{i}}\right)^{-1} \beta_{2}}\right)^{\alpha_{2}-\mathrm{nu}}}\right)}
$$

$$
\begin{aligned}
& \operatorname{Var}\left(\hat{\mathrm{u}}_{2}{ }^{*} / \mathrm{X}\right)=
\end{aligned}
$$

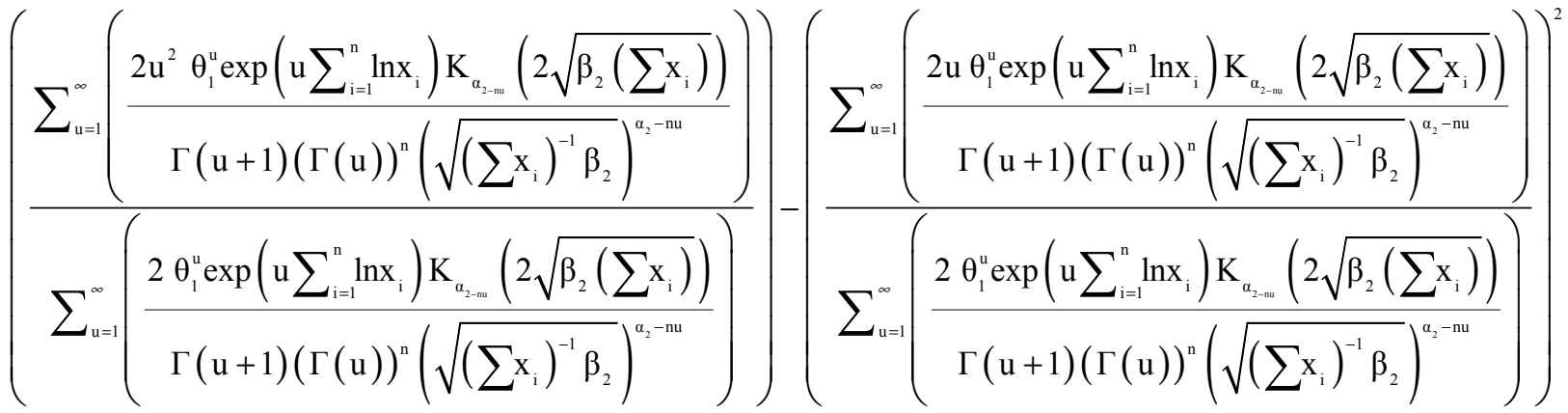




$$
\begin{aligned}
& \operatorname{Var}\left(\hat{\mathrm{v}}_{2}{ }^{*} / \mathrm{X}\right)=
\end{aligned}
$$

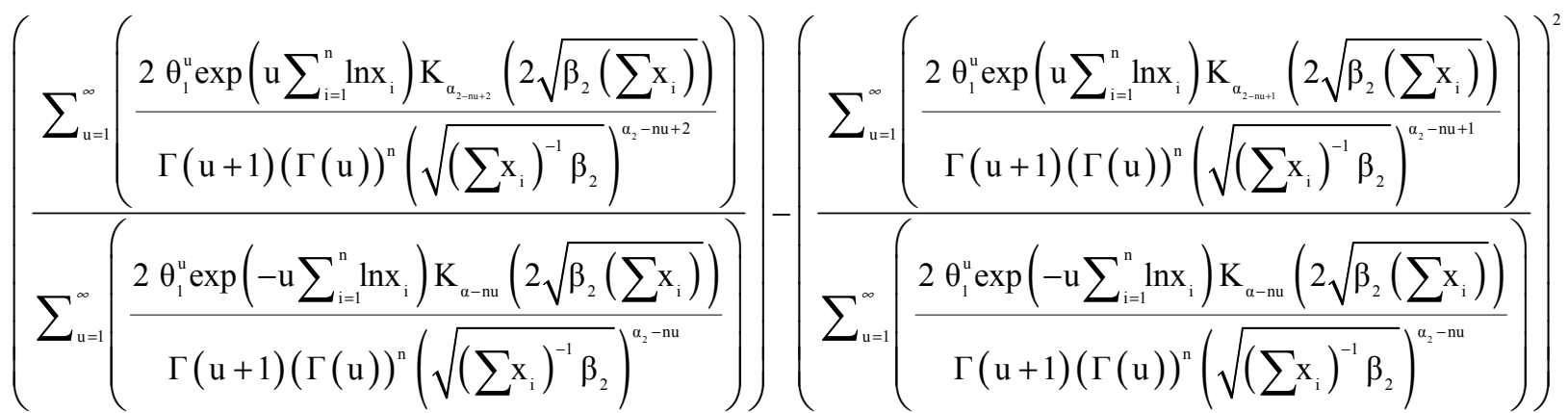

Posterior Distribution under Generalized Truncated Geometric and Inverted Gamma Priors

If the prior assumed for shape parameter $\mathrm{u}$ is the generalized truncated geometric distribution then the pdf is given by

$$
\begin{aligned}
& \mathrm{g}_{31}\left(\mathrm{u}, \theta_{2}\right)=\left(1-\mathrm{m}+\theta_{2}\right)\left(\begin{array}{c}
\mathrm{mu}-1 \\
\mathrm{u}-1
\end{array}\right)\left(1-\theta_{2}\right)^{\mathrm{u}-1} \theta_{2}^{\mathrm{mu}-\mathrm{u}} \\
& \mathrm{u}=1,2,3, \ldots ; 0<\theta_{2}<1
\end{aligned}
$$

The prior for scale parameter $\mathrm{v}$ is assumed to be an inverted Gamma distribution having pdf

$$
\begin{aligned}
& \mathrm{g}_{12}\left(\mathrm{v} ; \alpha_{1}, \beta_{1}\right)=\frac{\beta_{1}^{\alpha_{1}} \mathrm{v}^{-\left(\alpha_{1}+1\right)} \exp \left(-\mathrm{v}^{-1} \beta_{1}\right)}{\Gamma\left(\alpha_{1}\right)} \\
& \mathrm{v}>1, \alpha_{1}>0, \beta_{1}>0
\end{aligned}
$$

and the joint prior distribution of $\mathrm{u}$ and $\mathrm{v}$ is defined as:

$$
\mathrm{g}_{3}(\mathrm{u}, \mathrm{v} / \mathrm{X})=\mathrm{g}_{31}\left(\mathrm{u}, \theta_{2}\right) \cdot \mathrm{g}_{12}\left(\mathrm{v} ; \alpha_{1}, \beta_{1}\right) .
$$

By combining likelihood function (3.1) and joint prior function (8.3), the joint posterior distribution of $u$ and $v$ is (3.2.3.4). The marginal posterior distributions of $\mathrm{u}$ and $\mathrm{v}$ are (3.2.3.5) and (3.2.3.6).The expression for Bayes estimators $\mathrm{u}$ and $\mathrm{v}$ under the squared error loss function with their respective posterior variance are (3.2.3.7), (3.2.3.8), (3.2.3.9) and (3.2.3.10). 


$$
\begin{aligned}
& \underline{\left(\begin{array}{c}
m u-1 \\
u-1
\end{array}\right)\left(1-\theta_{2}\right)^{u-1} \theta_{2}^{m u-u} \exp \left(u \sum_{i=1}^{n} \operatorname{lnx}_{i}\right) v^{-\left(\alpha_{1}+n u+1\right)} \exp \left\{-v^{-1}\left(\beta_{1}+\sum_{i=1}^{n} x_{i}\right\}\right.} \\
& g_{3}(u, v / X)=\frac{(\Gamma(u))^{n}}{\sum_{u=1}^{\infty}\left(\frac{\left(\begin{array}{c}
m u-1 \\
u-1
\end{array}\right)\left(1-\theta_{2}\right)^{u-1} \theta_{2}^{m u-u} \exp \left(u \sum_{i=1}^{n} \ln x_{i}\right) \int_{0}^{\infty} v^{-\left(a_{1}+n u+1\right)} \exp \left\{-v^{-1}\left(\beta_{1}+\sum_{i=1}^{n} x_{i}\right)\right\} d b}{(\Gamma(u))^{n}}\right)} \\
& \underline{\left(\begin{array}{c}
m u-1 \\
u-1
\end{array}\right)\left(1-\theta_{2}\right)^{u-1} \theta_{2}^{m u-u} \exp \left(u \sum_{i=1}^{n} \operatorname{lnx}_{i}\right) v^{-\left(a_{1}+n u+1\right)} \exp \left\{-v^{-1}\left(\beta_{1}+\sum_{i=1}^{n} x_{i}\right\}\right.} \\
& g_{3}(u, v / X)=\frac{(\Gamma(u))^{n}}{\sum_{u=1}^{\infty}\left(\frac{\left(\begin{array}{c}
m u-1 \\
u-1
\end{array}\right)\left(1-\theta_{2}\right)^{u-1} \theta_{2}^{m u-u} \exp \left(u \sum_{i=1}^{n} \ln x_{i}\right) \Gamma\left(\alpha_{1}+n u\right)}{(\Gamma(u))^{n}\left(\beta_{1}+\sum_{i=1}^{n} x_{i}\right)^{\alpha_{1}+n u}}\right)}
\end{aligned}
$$

$$
\begin{gathered}
\left.\mathrm{g}_{3}(\mathrm{u} / \mathrm{X})=\frac{\left(\begin{array}{c}
\mathrm{mu}-1 \\
\mathrm{u}-1
\end{array}\right)\left(1-\theta_{2}\right)^{\mathrm{ul}} \theta_{2}^{\mathrm{mu}-\mathrm{u}} \exp \left(\mathrm{u} \sum_{\mathrm{i}=1}^{\mathrm{n}} \ln \mathrm{x}_{\mathrm{i}}\right) \int_{0}^{\infty} \mathrm{v}^{-\left(\alpha_{1}+\mathrm{nu}+1\right)} \exp \left\{-\mathrm{v}^{-1}\left(\beta_{1}+\sum_{\mathrm{i}=1}^{\mathrm{n}} \mathrm{x}_{\mathrm{i}}\right)\right\} \mathrm{db}}{(\Gamma(\mathrm{u}))^{\mathrm{n}}}\right) \\
\sum_{\mathrm{u}=1}^{\infty}\left(\frac{\left(\begin{array}{c}
\mathrm{mu}-1 \\
\mathrm{u}-1
\end{array}\right)\left(1-\theta_{2}\right)^{\mathrm{u}-1} \theta_{2}^{\mathrm{mu}-\mathrm{u}} \exp \left(\mathrm{u} \sum_{\mathrm{i}=1}^{\mathrm{n}} \ln \mathrm{x}_{\mathrm{i}}\right) \Gamma\left(\alpha_{1}+\mathrm{nu}\right)}{(\Gamma(\mathrm{u}))^{\mathrm{n}}\left(\beta_{1}+\sum_{\mathrm{i}=1}^{\mathrm{n}} \mathrm{x}_{\mathrm{i}}\right)^{\alpha_{1}+\mathrm{nu}}}\right) \\
\mathrm{g}_{3}(\mathrm{u} / \mathrm{X})=\frac{\left(\begin{array}{c}
\mathrm{mu}-1 \\
\mathrm{u}-1
\end{array}\right)\left(1-\theta_{2}\right)^{\mathrm{u}-1} \theta_{2}^{\mathrm{mu}-\mathrm{u}} \exp \left(\mathrm{u} \sum_{\mathrm{i}=1}^{\mathrm{n}} \ln \mathrm{x}_{\mathrm{i}}\right) \Gamma\left(\alpha_{1}+\mathrm{nu}\right)}{(\Gamma(\mathrm{u}))^{\mathrm{n}}\left(\beta_{1}+\sum_{\mathrm{i}=1}^{\mathrm{n}} \mathrm{x}_{\mathrm{i}}\right)^{\alpha_{1}+\mathrm{nu}}}
\end{gathered}
$$


BAYESIAN ESTIMATION OF ERLANG DISTRIBUTION UNDER DIFFERENT PRIORS

$\mathrm{g}_{3}(\mathrm{v} / \mathrm{X})=\frac{\sum_{\mathrm{u}=1}^{\infty}\left(\frac{\left(\begin{array}{c}\mathrm{mu}-1 \\ \mathrm{u}-1\end{array}\right)\left(1-\theta_{2}\right)^{\mathrm{u}-1} \theta_{2}^{\mathrm{mu}-\mathrm{u}} \exp \left(\mathrm{u} \sum_{\mathrm{i}=1}^{\mathrm{n}} \ln \mathrm{x}_{\mathrm{i}}\right) \mathrm{v}^{-\mathrm{nu}}}{(\Gamma(\mathrm{u}))^{\mathrm{n}}}\right) \frac{\exp \left\{-\mathrm{v}^{-1}\left(\beta_{1}+\sum_{\mathrm{i}=1}^{\mathrm{n}} \mathrm{x}_{\mathrm{i}}\right\}\right.}{\mathrm{v}^{\alpha_{1}+1}}}{\sum_{\mathrm{u}=1}^{\infty}\left(\frac{\left(\begin{array}{c}\mathrm{mu}-1 \\ \mathrm{u}-1\end{array}\right)\left(1-\theta_{2}\right)^{\mathrm{u}-1} \theta_{2}^{\mathrm{mu}-\mathrm{u}} \exp \left(\mathrm{u} \sum_{\mathrm{i}=1}^{\mathrm{n}} \ln \mathrm{x}_{\mathrm{i}}\right) \Gamma\left(\alpha_{1}+\mathrm{nu}\right)}{(\Gamma(\mathrm{u}))^{\mathrm{n}}\left(\beta_{1}+\sum_{\mathrm{i}=1}^{\mathrm{n}} \mathrm{x}_{\mathrm{i}}\right)^{\alpha_{1}+\mathrm{nu}}}\right)} ; \mathrm{v}>0$

$\hat{\mathrm{u}}_{3} / \mathrm{X}=\frac{\sum_{\mathrm{u}=1}^{\infty}\left(\frac{\mathrm{u}\left(\begin{array}{c}\mathrm{mu}-1 \\ \mathrm{u}-1\end{array}\right)\left(1-\theta_{2}\right)^{\mathrm{u}-1} \theta_{2}^{\mathrm{mu}-\mathrm{u}} \exp \left(\mathrm{u} \sum_{\mathrm{i}=1}^{\mathrm{n}} \ln \mathrm{x}_{\mathrm{i}}\right) \Gamma\left(\alpha_{1}+\mathrm{nu}\right)}{(\Gamma(\mathrm{u}))^{\mathrm{n}}\left(\beta_{1}+\sum_{\mathrm{i}=1}^{\mathrm{n}} \mathrm{x}_{\mathrm{i}}\right)^{\alpha_{1}+\mathrm{nu}}}\right)}{\sum_{\mathrm{u}=1}^{\infty}\left(\frac{\left(\begin{array}{c}\mathrm{mu}-1 \\ \mathrm{u}-1\end{array}\right)\left(1-\theta_{2}\right)^{\mathrm{u}-1} \theta_{2}^{\mathrm{mu}-\mathrm{u}} \exp \left(\mathrm{u} \sum_{\mathrm{i}=1}^{\mathrm{n}} \ln \mathrm{x}_{\mathrm{i}}\right) \Gamma\left(\alpha_{1}+n u\right)}{(\Gamma(\mathrm{u}))^{\mathrm{n}}\left(\beta_{1}+\sum_{\mathrm{i}=1}^{\mathrm{n}} \mathrm{x}_{\mathrm{i}}\right)^{\alpha_{1}+\mathrm{nu}}}\right)}$

$\hat{\mathrm{v}}_{3 / \mathrm{X}}=\frac{\sum_{\mathrm{u}=1}^{\infty}\left(\frac{\left(\begin{array}{c}\mathrm{mu}-1 \\ \mathrm{u}-1\end{array}\right)\left(1-\theta_{2}\right)^{\mathrm{u}-1} \theta_{2}^{\mathrm{mu}-\mathrm{u}} \exp \left(\mathrm{u} \sum_{\mathrm{i}=1}^{\mathrm{n}} \ln \mathrm{x}_{\mathrm{i}}\right) \int_{\mathrm{o}}^{\infty} \mathrm{v} \cdot \mathrm{v}^{-\left(\alpha_{1}+\mathrm{nu}+1\right)} \exp \left\{-\mathrm{v}^{-1}\left(\beta_{1}+\sum_{\mathrm{i}=1}^{\mathrm{n}} \mathrm{x}_{\mathrm{i}}\right)\right\} \mathrm{db}}{(\Gamma(\mathrm{u}))^{\mathrm{n}}}\right)}{\sum_{\mathrm{u}=1}^{\infty}\left(\frac{\left(\begin{array}{c}\mathrm{mu}-1 \\ \mathrm{u}-1\end{array}\right)\left(1-\theta_{2}\right)^{\mathrm{u}-1} \theta_{2}^{\mathrm{mu}-\mathrm{u}} \exp \left(\mathrm{u} \sum_{\mathrm{i}=1}^{\mathrm{n}} \ln \mathrm{x}_{\mathrm{i}}\right) \Gamma\left(\alpha_{1}+\mathrm{nu}\right)}{(\Gamma(\mathrm{u}))^{\mathrm{n}}\left(\beta_{1}+\sum_{\mathrm{i}=1}^{\mathrm{n}} \mathrm{x}_{\mathrm{i}}\right)^{\alpha_{1}+\mathrm{nu}}}\right)}$

$\hat{\mathrm{v}}_{3} / \mathrm{X}=\frac{\sum_{\mathrm{u}=1}^{\infty}\left(\frac{\left(\begin{array}{c}\mathrm{mu}-1 \\ \mathrm{u}-1\end{array}\right)\left(1-\theta_{2}\right)^{\mathrm{u}-1} \theta_{2}^{\mathrm{mu}-\mathrm{u}} \exp \left(\mathrm{u} \sum_{\mathrm{i}=1}^{\mathrm{n}} \ln \mathrm{x}_{\mathrm{i}}\right) \Gamma\left(\alpha_{1}+\mathrm{nu}-1\right)}{(\Gamma(\mathrm{u}))^{\mathrm{n}}\left(\beta_{1}+\sum_{\mathrm{i}=1}^{\mathrm{n}} \mathrm{x}_{\mathrm{i}}\right)^{\alpha_{1}+\mathrm{nu}-1}}\right)}{\sum_{\mathrm{u}=1}^{\infty}\left(\frac{\left(\begin{array}{c}\mathrm{mu}-1 \\ \mathrm{u}-1\end{array}\right)\left(1-\theta_{2}\right)^{\mathrm{u}-1} \theta_{2}^{\mathrm{mu}-\mathrm{u}} \exp \left(\mathrm{u} \sum_{\mathrm{i}=1}^{\mathrm{n}} \ln \mathrm{x}_{\mathrm{i}}\right) \Gamma\left(\alpha_{1}+\mathrm{nu}\right)}{(\Gamma(\mathrm{u}))^{\mathrm{n}}\left(\beta_{1}+\sum_{\mathrm{i}=1}^{\mathrm{n}} \mathrm{x}_{\mathrm{i}}\right)^{\alpha_{1}+\mathrm{nu}}}\right)}$ 


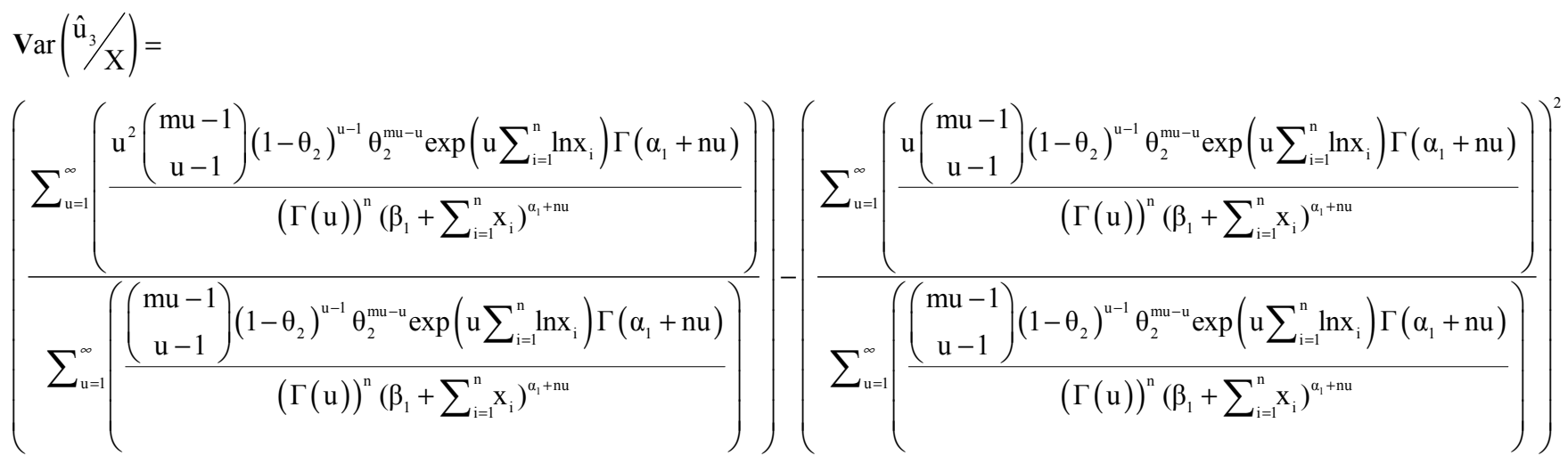

$$
\begin{aligned}
& \operatorname{Var}\left(\hat{\mathrm{v}}_{\mathrm{s}} / \mathrm{X}\right)=\left(\frac{\sum_{\mathrm{u}=1}^{\infty}\left(\frac{\left(\begin{array}{c}
\mathrm{mu}-1 \\
\mathrm{u}-1
\end{array}\right)\left(1-\theta_{2}\right)^{\mathrm{u}-1} \theta_{2}^{\mathrm{mu}-\mathrm{u}} \exp \left(\mathrm{u} \sum_{\mathrm{i}=1}^{\mathrm{n}} \ln \mathrm{x}_{\mathrm{i}}\right) \int_{\mathrm{o}}^{\infty} \mathrm{v}^{2} \cdot \mathrm{v}^{-\left(\alpha_{1}+\mathrm{nu}+1\right)} \exp \left\{-\mathrm{v}^{-1}\left(\beta_{1}+\sum_{\mathrm{i}=1}^{\mathrm{n}} \mathrm{x}_{\mathrm{i}}\right)\right\} \mathrm{db}}{(\Gamma(\mathrm{u}))^{\mathrm{n}}}\right)}{\sum_{\mathrm{u}=1}^{\infty}\left(\frac{\left(\begin{array}{c}
\mathrm{mu}-1 \\
\mathrm{u}-1
\end{array}\right)\left(1-\theta_{2}\right)^{\mathrm{u}-1} \theta_{2}^{\mathrm{mu}-\mathrm{u}} \exp \left(\mathrm{u} \sum_{\mathrm{i}=1}^{\mathrm{n}} \ln \mathrm{x}_{\mathrm{i}}\right) \Gamma\left(\alpha_{1}+\mathrm{nu}\right)}{(\Gamma(\mathrm{u}))^{\mathrm{n}}\left(\beta_{1}+\sum_{\mathrm{i}=1}^{\mathrm{n}} \mathrm{x}_{\mathrm{i}}\right)^{\alpha_{1}+\mathrm{nu}}}\right)}\right) \\
& -\left(\frac{\sum_{u=1}^{\infty}\left(\frac{\left(\begin{array}{c}
m u-1 \\
u-1
\end{array}\right)\left(1-\theta_{2}\right)^{u-1} \theta_{2}^{m u-u} \exp \left(u \sum_{i=1}^{n} \ln x_{i}\right) \Gamma\left(\alpha_{1}+n u-1\right)}{(\Gamma(u))^{n}\left(\beta_{1}+\sum_{i=1}^{n} x_{i}\right)^{\alpha_{1}+n u-1}}\right)}{\sum_{u=1}^{\infty}\left(\frac{\left(\begin{array}{c}
m u-1 \\
u-1
\end{array}\right)\left(1-\theta_{2}\right)^{u-1} \theta_{2}^{m u-u} \exp \left(u \sum_{i=1}^{n} \ln x_{i}\right) \Gamma\left(\alpha_{1}+n u\right)}{(\Gamma(u))^{n}\left(\beta_{1}+\sum_{i=1}^{n} x_{i}\right)^{\alpha_{1}+n u}}\right)}\right)^{2}
\end{aligned}
$$




$$
\begin{aligned}
& \left.\operatorname{Var}\left(\hat{\mathrm{v}}_{3} / \mathrm{X}\right)=\left(\frac{\sum_{\mathrm{u}=1}^{\infty}\left(\frac{\left(\begin{array}{c}
\mathrm{mu}-1 \\
\mathrm{u}-1
\end{array}\right)\left(1-\theta_{2}\right)^{\mathrm{u}-1} \theta_{2}^{\mathrm{mu}-\mathrm{u}} \exp \left(\mathrm{u} \sum_{\mathrm{i}=1}^{\mathrm{n}} \ln \mathrm{x}_{\mathrm{i}}\right) \Gamma\left(\alpha_{1}+\mathrm{nu}-2\right)}{(\Gamma(\mathrm{u}))^{\mathrm{n}}\left(\beta_{1}+\sum_{\mathrm{i}=1}^{\mathrm{n}} \mathrm{x}_{\mathrm{i}}\right)^{\alpha_{1}+\mathrm{nu}-2}}\right)}{\sum_{\mathrm{u}=1}^{\infty}\left(\frac{\left(\begin{array}{c}
\mathrm{mu}-1 \\
\mathrm{u}-1
\end{array}\right)\left(1-\theta_{2}\right)^{\mathrm{u}-1} \theta_{2}^{\mathrm{mu}-\mathrm{u}} \exp \left(\mathrm{u} \sum_{\mathrm{i}=1}^{\mathrm{n}} \ln \mathrm{x}_{\mathrm{i}}\right) \Gamma\left(\alpha_{1}+\mathrm{nu}\right)}{(\Gamma(\mathrm{u}))^{\mathrm{n}}\left(\beta_{1}+\sum_{\mathrm{i}=1}^{\mathrm{n}} \mathrm{x}_{\mathrm{i}}\right)^{\alpha_{1}+\mathrm{nu}}}\right)}\right)\right) \\
& \left.-\left(\frac{\sum_{\mathrm{u}=1}^{\infty}\left(\frac{\left(\begin{array}{c}
\mathrm{mu}-1 \\
\mathrm{u}-1
\end{array}\right)\left(1-\theta_{2}\right)^{\mathrm{u}-1} \theta_{2}^{\mathrm{mu}-\mathrm{u}} \exp \left(\mathrm{u} \sum_{\mathrm{i}=1}^{\mathrm{n}} \ln \mathrm{x}_{\mathrm{i}}\right) \Gamma\left(\alpha_{1}+\mathrm{nu}-1\right)}{(\Gamma(\mathrm{u}))^{\mathrm{n}}\left(\beta_{1}+\sum_{\mathrm{i}=1}^{\mathrm{n}} \mathrm{x}_{\mathrm{i}}\right)^{\alpha_{1}+\mathrm{nu}-1}}\right)}{\sum_{\mathrm{u}=1}^{\infty}\left(\frac{\left(\begin{array}{c}
\mathrm{mu}-1 \\
\mathrm{u}-1
\end{array}\right)\left(1-\theta_{2}\right)^{\mathrm{u}-1} \theta_{2}^{\mathrm{mu}-\mathrm{u}} \exp \left(\mathrm{u} \sum_{\mathrm{i}=1}^{\mathrm{n}} \ln \mathrm{x}_{\mathrm{i}}\right) \Gamma\left(\alpha_{1}+\mathrm{nu}\right)}{(\Gamma(\mathrm{u}))^{\mathrm{n}}\left(\beta_{1}+\sum_{\mathrm{i}=1}^{\mathrm{n}} \mathrm{x}_{\mathrm{i}}\right)^{\alpha_{1}+\mathrm{nu}}}\right)}\right)^{2}\right)
\end{aligned}
$$

Special Cases

For $\mathrm{m}=1$, the generalized truncated geometric distribution reduces to a truncated geometric distribution, therefore, the Bayes estimator for scale parameter $u$ and shape parameter $\mathrm{v}$ with their respective posterior variance are given by

$$
\begin{aligned}
& \hat{\mathrm{u}}_{3}^{*} / \mathrm{X}= \\
& \frac{\sum_{\mathrm{u}=1}^{\infty}\left(\frac{\mathrm{u}\left(1-\theta_{2}\right)^{\mathrm{u}-1} \exp \left(\mathrm{u} \sum_{\mathrm{i}=1}^{\mathrm{n}} \ln \mathrm{x}_{\mathrm{i}}\right) \Gamma\left(\alpha_{1}+\mathrm{nu}\right)}{\left.(\Gamma(\mathrm{u}))^{\mathrm{n}}\left(\beta_{1}+\sum_{\mathrm{i}=1}^{\mathrm{n}} \mathrm{x}_{\mathrm{i}}\right)^{\alpha_{1}+\mathrm{nu}}\right)}\right.}{\sum_{\mathrm{u}=1}^{\infty}\left(\frac{\left(1-\theta_{2}\right)^{\mathrm{u}-1} \exp \left(\mathrm{u} \sum_{\mathrm{i}=1}^{\mathrm{n}} \ln \mathrm{x}_{\mathrm{i}}\right) \Gamma\left(\alpha_{1}+\mathrm{nu}\right)}{\left.(\Gamma(\mathrm{u}))^{\mathrm{n}}\left(\beta_{1}+\sum_{\mathrm{i}=1}^{\mathrm{n}} \mathrm{x}_{\mathrm{i}}\right)^{\alpha_{1}+\mathrm{nu}}\right)}\right.}
\end{aligned}
$$

and

$$
\begin{aligned}
& \hat{\mathrm{v}}_{3}^{*} / \mathrm{X}= \\
& \frac{\sum_{\mathrm{u}=1}^{\infty}\left(\frac{\left(1-\theta_{2}\right)^{\mathrm{u}-1} \exp \left(\mathrm{u} \sum_{\mathrm{i}=1}^{\mathrm{n}} \ln \mathrm{x}_{\mathrm{i}}\right) \Gamma\left(\alpha_{1}+\mathrm{nu}-1\right)}{\left.(\Gamma(\mathrm{u}))^{\mathrm{n}}\left(\beta_{1}+\sum_{\mathrm{i}=1}^{\mathrm{n}} \mathrm{x}_{\mathrm{i}}\right)^{\alpha_{1}+\mathrm{nu}-1}\right)}\right.}{\sum_{\mathrm{u}=1}^{\infty}\left(\frac{\left(1-\theta_{2}\right)^{\mathrm{u}-1} \exp \left(\mathrm{u} \sum_{\mathrm{i}=1}^{\mathrm{n}} \ln \mathrm{x}_{\mathrm{i}}\right) \Gamma\left(\alpha_{1}+\mathrm{nu}\right)}{(\Gamma(\mathrm{u}))^{\mathrm{n}}\left(\beta_{1}+\sum_{\mathrm{i}=1}^{\mathrm{n}} \mathrm{x}_{\mathrm{i}}\right)^{\alpha_{1}+\mathrm{nu}}}\right)}
\end{aligned}
$$

and in (3.2.3.13) and (3.2.3.14). 


$$
\begin{aligned}
\operatorname{Var}\left(\hat{\mathrm{u}}_{3}^{*} / \mathrm{X}\right)= & \left(\frac{\sum_{\mathrm{u}=1}^{\infty}\left(\frac{\mathrm{u}^{2}\left(1-\theta_{2}\right)^{\mathrm{u}-1} \exp \left(\mathrm{u} \sum_{\mathrm{i}=1}^{\mathrm{n}} \ln \mathrm{x}_{\mathrm{i}}\right) \Gamma\left(\alpha_{1}+\mathrm{nu}\right)}{(\Gamma(\mathrm{u}))^{\mathrm{n}}\left(\beta_{1}+\sum_{\mathrm{i}=1}^{\mathrm{n}} \mathrm{x}_{\mathrm{i}}\right)^{\alpha_{1}+\mathrm{nu}}}\right)}{\sum_{\mathrm{u}=1}^{\infty}\left(\frac{\left(1-\theta_{2}\right)^{\mathrm{u}-1} \exp \left(\mathrm{u} \sum_{\mathrm{i}=1}^{\mathrm{n}} \ln \mathrm{x}_{\mathrm{i}}\right) \Gamma\left(\alpha_{1}+\mathrm{nu}\right)}{(\Gamma(\mathrm{u}))^{\mathrm{n}}\left(\beta_{1}+\sum_{\mathrm{i}=1}^{\mathrm{n}} \mathrm{x}_{\mathrm{i}}\right)^{\alpha_{1}+\mathrm{nu}}}\right)}\right) \\
& -\left(\frac{\sum_{\mathrm{u}=1}^{\infty}\left(\frac{\mathrm{u}\left(1-\theta_{2}\right)^{\mathrm{u}-1} \exp \left(\mathrm{u} \sum_{\mathrm{i}=1}^{\mathrm{n}} \ln \mathrm{x}_{\mathrm{i}}\right) \Gamma\left(\alpha_{1}+\mathrm{nu}\right)}{(\Gamma(\mathrm{u}))^{\mathrm{n}}\left(\beta_{1}+\sum_{\mathrm{i}=1}^{\mathrm{n}} \mathrm{x}_{\mathrm{i}}\right)^{\alpha_{1}+\mathrm{nu}}}\right)}{\sum_{\mathrm{u}=1}^{\infty}\left(\frac{\left(1-\theta_{2}\right)^{\mathrm{u}-1} \exp \left(\mathrm{u} \sum_{\mathrm{i}=1}^{\mathrm{n}} \ln \mathrm{x}_{\mathrm{i}}\right) \Gamma\left(\alpha_{1}+\mathrm{nu}\right)}{(\Gamma(\mathrm{u}))^{\mathrm{n}}\left(\beta_{1}+\sum_{\mathrm{i}=1}^{\mathrm{n}} \mathrm{x}_{\mathrm{i}}\right)^{\alpha_{1}+\mathrm{nu}}}\right)}\right)^{2}
\end{aligned}
$$

$$
\begin{aligned}
\operatorname{Var}\left(\hat{\mathrm{v}}_{3}^{*} / \mathrm{X}\right)= & \left(\frac{\sum_{\mathrm{u}=1}^{\infty}\left(\frac{\left(1-\theta_{2}\right)^{\mathrm{u}-1} \exp \left(\mathrm{u} \sum_{\mathrm{i}=1}^{\mathrm{n}} \ln \mathrm{x}_{\mathrm{i}}\right) \Gamma\left(\alpha_{1}+\mathrm{nu}-2\right)}{(\Gamma(\mathrm{u}))^{\mathrm{n}}\left(\beta_{1}+\sum_{\mathrm{i}=1}^{\mathrm{n}} \mathrm{x}_{\mathrm{i}}\right)^{\alpha_{1}+\mathrm{nu}-2}}\right)}{\sum_{\mathrm{u}=1}^{\infty}\left(\frac{\left(1-\theta_{2}\right)^{\mathrm{u}-1} \exp \left(\mathrm{u} \sum_{\mathrm{i}=1}^{\mathrm{n}} \ln \mathrm{x}_{\mathrm{i}}\right) \Gamma\left(\alpha_{1}+\mathrm{nu}\right)}{(\Gamma(\mathrm{u}))^{\mathrm{n}}\left(\beta_{1}+\sum_{\mathrm{i}=1}^{\mathrm{n}} \mathrm{x}_{\mathrm{i}}\right)^{\alpha_{1}+\mathrm{nu}}}\right)}\right) \\
& -\left(\frac{\sum_{\mathrm{u}=1}^{\infty}\left(\frac{\left(1-\theta_{2}\right)^{\mathrm{u}-1} \exp \left(\mathrm{u} \sum_{\mathrm{i}=1}^{\mathrm{n}} \ln \mathrm{x}_{\mathrm{i}}\right) \Gamma\left(\alpha_{1}+\mathrm{nu}-1\right)}{(\Gamma(\mathrm{u}))^{\mathrm{n}}\left(\beta_{1}+\sum_{\mathrm{i}=1}^{\mathrm{n}} \mathrm{x}_{\mathrm{i}}\right)^{\alpha_{1}+n u-1}}\right)}{\sum_{\mathrm{u}=1}^{\infty}\left(\frac{\left(1-\theta_{2}\right)^{\mathrm{u}-1} \exp \left(\mathrm{u} \sum_{\mathrm{i}=1}^{\mathrm{n}} \ln \mathrm{ln}_{\mathrm{i}}\right) \Gamma\left(\alpha_{1}+\mathrm{nu}\right)}{(\Gamma(\mathrm{u}))^{\mathrm{n}}\left(\beta_{1}+\sum_{\mathrm{i}=1}^{\mathrm{n}} \mathrm{x}_{\mathrm{i}}\right)^{\alpha_{1}+\mathrm{nu}}}\right)}\right)^{2}
\end{aligned}
$$




\section{BAYESIAN ESTIMATION OF ERLANG DISTRIBUTION UNDER DIFFERENT PRIORS}

Posterior Distribution under Generalized Truncated Geometric and Gamma Priors

If the prior assumed for shape parameter

$\mathrm{u}$ is the generalized truncated geometric distribution, then the pdf is

$\mathrm{g}_{31}\left(\mathrm{u}, \theta_{2}\right)=\left(1-\mathrm{m}+\theta_{2}\right)\left(\begin{array}{c}\mathrm{mu}-1 \\ \mathrm{u}-1\end{array}\right)\left(1-\theta_{2}\right)^{\mathrm{u}-1} \theta_{2}^{\mathrm{mu}-\mathrm{u}} ;$

$\mathrm{u}=1,2,3, \ldots \ldots ; 0<\theta_{2}<1$

and the prior for scale parameter $\mathrm{v}$ is assumed to be a Gamma distribution with pdf

$$
\mathrm{g}_{21}\left(\mathrm{v} ; \alpha_{2}, \beta_{2}\right)=\frac{\beta_{2}^{\alpha_{2}} \mathrm{v}^{\alpha_{2}-1} \exp \left(-\mathrm{v} \beta_{2}\right)}{\Gamma\left(\alpha_{2}\right)} ;
$$$$
\mathrm{v}>1, \alpha_{2}>0, \beta_{2}>0 \text {. }
$$

The joint prior of $\mathrm{u}$ and $\mathrm{v}$ is defined as:

$$
\mathrm{g}_{4}(\mathrm{u}, \mathrm{v})=\mathrm{g}_{31}\left(\mathrm{u}, \theta_{2}\right) \cdot \mathrm{g}_{21}\left(\mathrm{v} ; \alpha_{2}, \beta_{2}\right) .
$$

By combining likelihood function (3.1) with joint prior function (9.3), the joint posterior distribution of $u$ and $v$ is given by (3.2.4.4). The marginal posterior distribution of $\mathrm{u}$ and $\mathrm{v}$ are given by (3.2.4.5) and (3.2.4.6). The expression for Bayes estimators for $\mathrm{u}$ and $\mathrm{v}$ with their respective posterior variance are given by (3.2.4.7), (3.2.4.8), (3.2.4.9) and (3.2.4.10).

$$
\begin{aligned}
& \left(\begin{array}{c}
\mathrm{mu}-1 \\
\mathrm{u}-1
\end{array}\right)\left(1-\theta_{2}\right)^{\mathrm{u}-1} \theta_{2}^{\mathrm{mu}-\mathrm{u}} \mathrm{v}^{\left(\alpha_{2}-\mathrm{nu}\right)-1} \exp \left\{\frac{-1}{2}\left(\left(2 \beta_{2}\right) \mathrm{v}+\frac{\left(2 \sum_{\mathrm{i}=1}^{\mathrm{n}} \mathrm{x}_{\mathrm{i}}\right)}{\mathrm{v}}\right)\right\} \\
& \mathrm{g}_{4}(\mathrm{u}, \mathrm{v} / \mathrm{X})=\frac{(\Gamma(\mathrm{u}))^{\mathrm{n}} \exp \left(-\mathrm{u} \sum_{\mathrm{i}=1}^{\mathrm{n}} \ln \mathrm{x}_{\mathrm{i}}\right)}{\left.\sum_{\mathrm{u}=1}^{\infty}\left(\frac{\left(\begin{array}{c}
\mathrm{mu}-1 \\
\mathrm{u}-1
\end{array}\right)\left(1-\theta_{2}\right)^{\mathrm{u}-1} \theta_{2}^{\mathrm{mu}-\mathrm{u}}}{(\Gamma(\mathrm{u}))^{\mathrm{n}} \exp \left(-\mathrm{u} \sum_{\mathrm{i}=1}^{\mathrm{n}} \ln \mathrm{x}_{\mathrm{i}}\right)} \int_{\mathrm{o}}^{\infty} \mathrm{v}^{\left(\alpha_{2}-\mathrm{nu}\right)-1} \exp \frac{-1}{2}\left(\left(2 \beta_{2}\right) \mathrm{v}+\frac{\left(2 \sum_{\mathrm{i}=1}^{\mathrm{n}} \mathrm{x}_{\mathrm{i}}\right)}{\mathrm{v}}\right)\right\} \mathrm{db}\right)} \\
& \left(\begin{array}{c}
m u-1 \\
\mathrm{u}-1
\end{array}\right)\left(1-\theta_{2}\right)^{\mathrm{u}-1} \theta_{2}^{\mathrm{mu}-\mathrm{u}} \mathrm{v}^{\left(\alpha_{2}-\mathrm{nu}\right)-1} \exp \left\{\frac{-1}{2}\left(\left(2 \beta_{2}\right) \mathrm{v}+\frac{\left(2 \sum_{\mathrm{i}=1}^{\mathrm{n}} \mathrm{x}_{\mathrm{i}}\right)}{\mathrm{v}}\right)\right\} \\
& \mathrm{g}_{4}(\mathrm{u}, \mathrm{v} / \mathrm{X})=\frac{(\Gamma(\mathrm{u}))^{\mathrm{n}} \exp \left(-\mathrm{u} \sum_{\mathrm{i}=1}^{\mathrm{n}} \ln \mathrm{x}_{\mathrm{i}}\right)}{\sum_{\mathrm{u}=1}^{\infty}\left(\frac{2\left(\begin{array}{c}
\mathrm{mu}-1 \\
\mathrm{u}-1
\end{array}\right)\left(1-\theta_{2}\right)^{\mathrm{u}-1} \theta_{2}^{\mathrm{mu}-\mathrm{u}} \exp \left(\mathrm{u} \sum_{\mathrm{i}=1}^{\mathrm{n}} \ln \mathrm{x}_{\mathrm{i}}\right) \mathrm{K}_{\alpha_{2}-\mathrm{nu}}\left(2 \sqrt{\beta_{2}\left(\sum \mathrm{x}_{\mathrm{i}}\right)}\right)}{(\Gamma(\mathrm{u}))^{\mathrm{n}}\left(\left(\sum \mathrm{x}_{\mathrm{i}}\right)^{-1} \beta_{2}\right)^{\alpha_{2}-\mathrm{nu}}}\right)}
\end{aligned}
$$




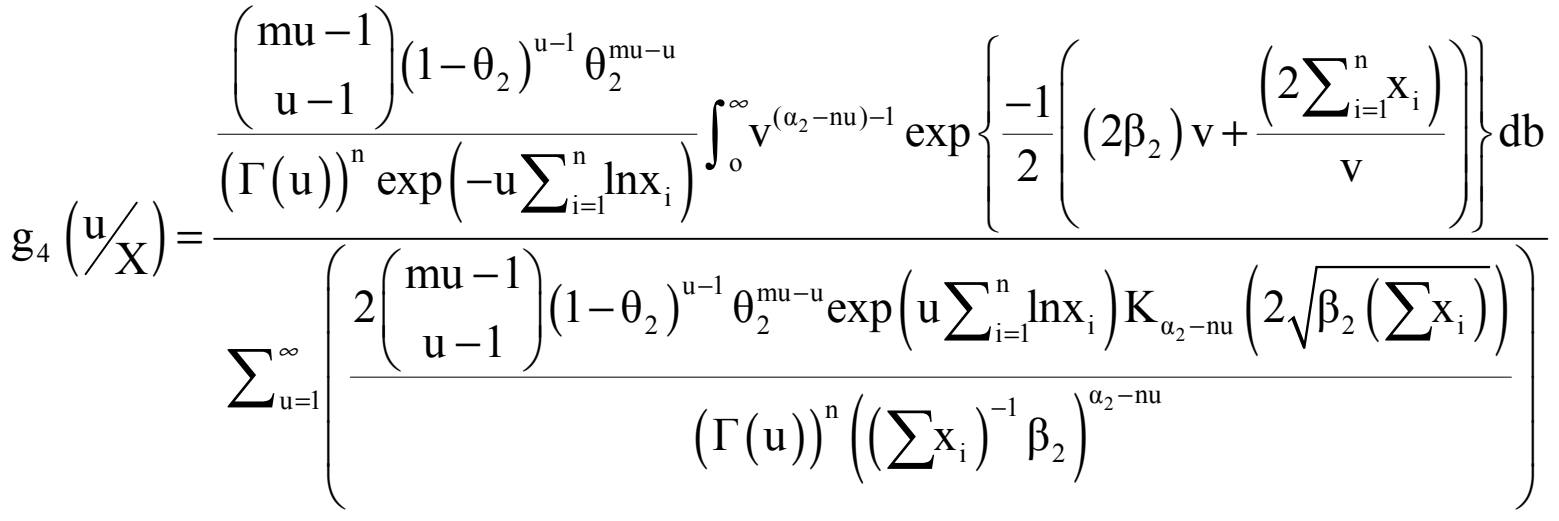

$$
\begin{aligned}
& \frac{2\left(\begin{array}{c}
\mathrm{mu}-1 \\
\mathrm{u}-1
\end{array}\right)\left(1-\theta_{2}\right)^{\mathrm{u}-1} \theta_{2}^{\mathrm{mu}-\mathrm{u}} \exp \left(\mathrm{u} \sum_{\mathrm{i}=1}^{\mathrm{n}} \ln \mathrm{x}_{\mathrm{i}}\right) \mathrm{K}_{\mathrm{a}_{2}-\mathrm{nu}}\left(2 \sqrt{\beta_{2}\left(\sum \mathrm{x}_{\mathrm{i}}\right)}\right)}{(\Gamma(\mathrm{u}))^{\mathrm{n}}\left(\left(\sum \mathrm{x}_{\mathrm{i}}\right)^{-1} \beta_{2}\right)^{\alpha_{2}-\mathrm{nu}}}
\end{aligned}
$$

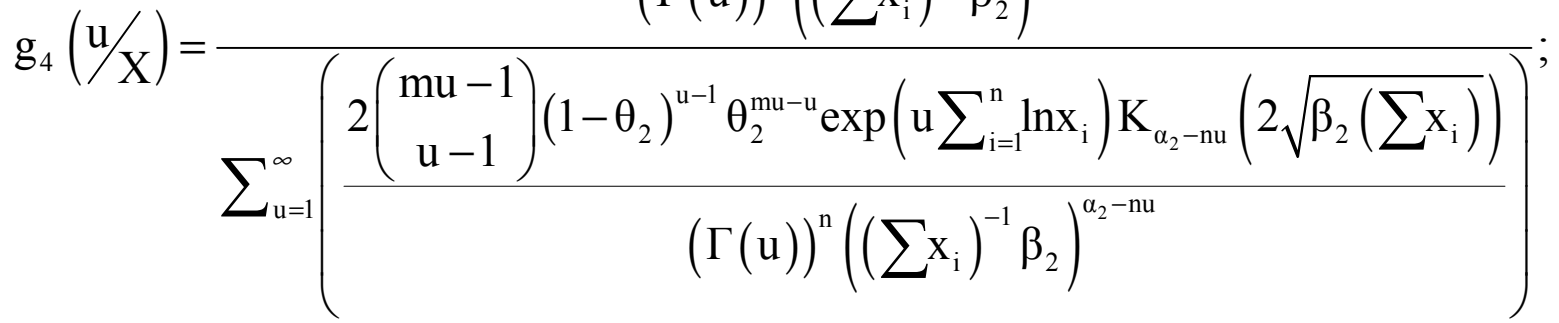

$\mathrm{u}=1,2,3, \ldots$

$\mathrm{g}_{4}(\mathrm{v} / \mathrm{X})=\frac{\sum_{\mathrm{u}=1}^{\infty}\left(\frac{\left(\begin{array}{c}\mathrm{mu}-1 \\ \mathrm{u}-1\end{array}\right)\left(1-\theta_{2}\right)^{\mathrm{u}-1} \theta_{2}^{\mathrm{mu}-\mathrm{u}} \mathrm{v}^{\left(\alpha_{2}-\mathrm{nu}\right)-1} \exp \left\{\frac{-1}{2}\left(\left(2 \beta_{2}\right) \mathrm{v}+\frac{\left(2 \sum_{\mathrm{i}=1}^{\mathrm{n}} \mathrm{x}_{\mathrm{i}}\right)}{\mathrm{v}}\right)\right.}{(\Gamma(\mathrm{u}))^{\mathrm{n}} \exp \left(-\mathrm{u} \sum_{\mathrm{i}=1}^{\mathrm{n}} \ln \mathrm{x}_{\mathrm{i}}\right)}\right)}{\sum_{\mathrm{u}=1}^{\infty}\left(\frac{2\left(\begin{array}{c}\mathrm{mu}-1 \\ \mathrm{u}-1\end{array}\right)\left(1-\theta_{2}\right)^{\mathrm{u}-1} \theta_{2}^{\mathrm{mu}-\mathrm{u}} \exp \left(\mathrm{u} \sum_{\mathrm{i}=1}^{\mathrm{n}} \ln \mathrm{x}_{\mathrm{i}}\right) \mathrm{K}_{\alpha_{2}-\mathrm{nu}}\left(2 \sqrt{\beta_{2}\left(\sum \mathrm{x}_{\mathrm{i}}\right)}\right)}{(\Gamma(\mathrm{u}))^{\mathrm{n}}\left(\left(\sum_{\mathrm{i}}\right)^{-1} \beta_{2}\right)^{\alpha_{2}-n u}}\right)} ; \mathrm{v}>0$ 


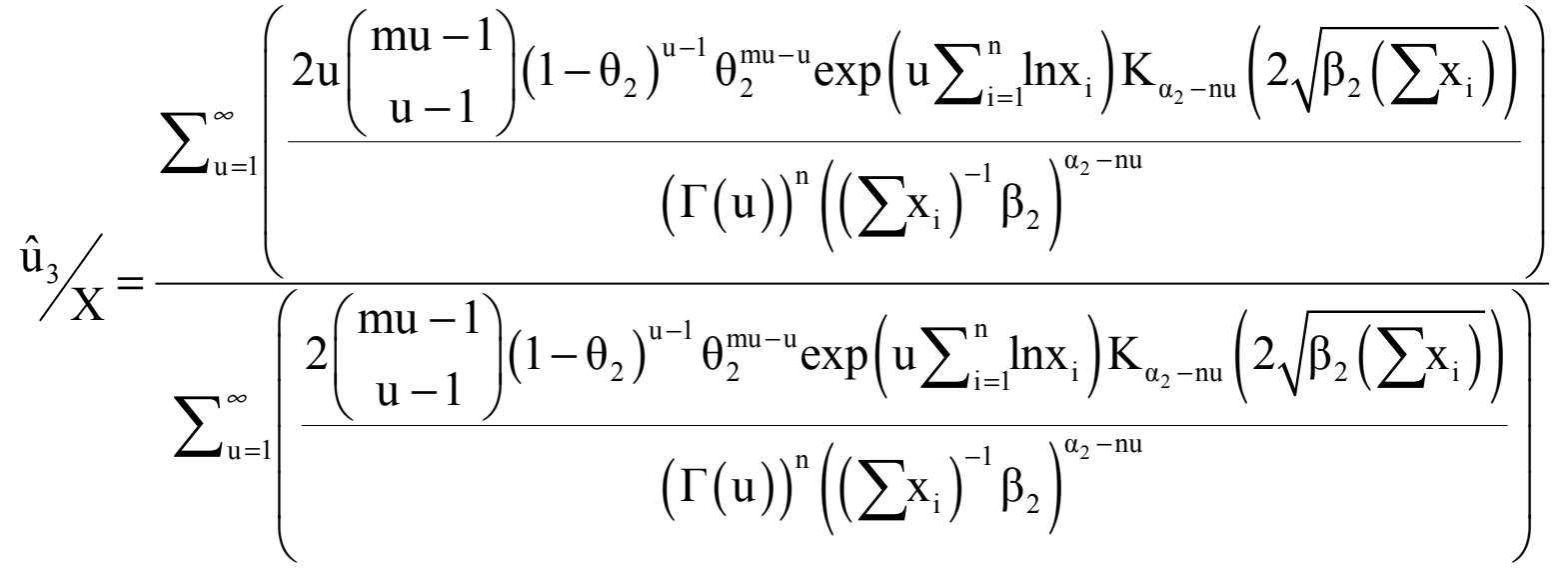

$$
\begin{aligned}
& \hat{\mathrm{v}}_{3} / \mathrm{X}=\frac{\left.\sum_{\mathrm{u}=1}^{\infty}\left(\frac{\left(\begin{array}{c}
\mathrm{mu}-1 \\
\mathrm{u}-1
\end{array}\right)\left(1-\theta_{2}\right)^{\mathrm{u}-1} \theta_{2}^{\mathrm{mu}-\mathrm{u}}}{(\Gamma(\mathrm{u}))^{\mathrm{n}} \exp \left(-\mathrm{u} \sum_{\mathrm{i}=1}^{\mathrm{n}} \ln \mathrm{x}_{\mathrm{i}}\right)} \int_{0}^{\infty} \mathrm{v} \cdot \mathrm{v}^{\left(\alpha_{2}-\mathrm{nu}\right)-1} \exp \left\{\frac{-1}{2}\left(2 \beta_{2}\right) \mathrm{v}+\frac{\left(2 \sum_{\mathrm{i}=1}^{\mathrm{n}} \mathrm{x}_{\mathrm{i}}\right)}{\mathrm{v}}\right)\right\} \mathrm{db}\right)}{\sum_{\mathrm{u}=1}^{\infty}\left(\frac{2\left(\begin{array}{c}
\mathrm{mu}-1 \\
\mathrm{u}-1
\end{array}\right)\left(1-\theta_{2}\right)^{\mathrm{u}-1} \theta_{2}^{\mathrm{mu}-\mathrm{u}} \exp \left(\mathrm{u} \sum_{\mathrm{i}=1}^{\mathrm{n}} \ln \mathrm{x}_{\mathrm{i}}\right) \mathrm{K}_{\alpha_{2}-\mathrm{nu}}\left(2 \sqrt{\beta_{2}\left(\sum \mathrm{x}_{\mathrm{i}}\right)}\right)}{(\Gamma(\mathrm{u}))^{\mathrm{n}}\left(\left(\sum \mathrm{x}_{\mathrm{i}}\right)^{-1} \beta_{2}\right)^{\alpha_{2}-\mathrm{nu}}}\right)}
\end{aligned}
$$

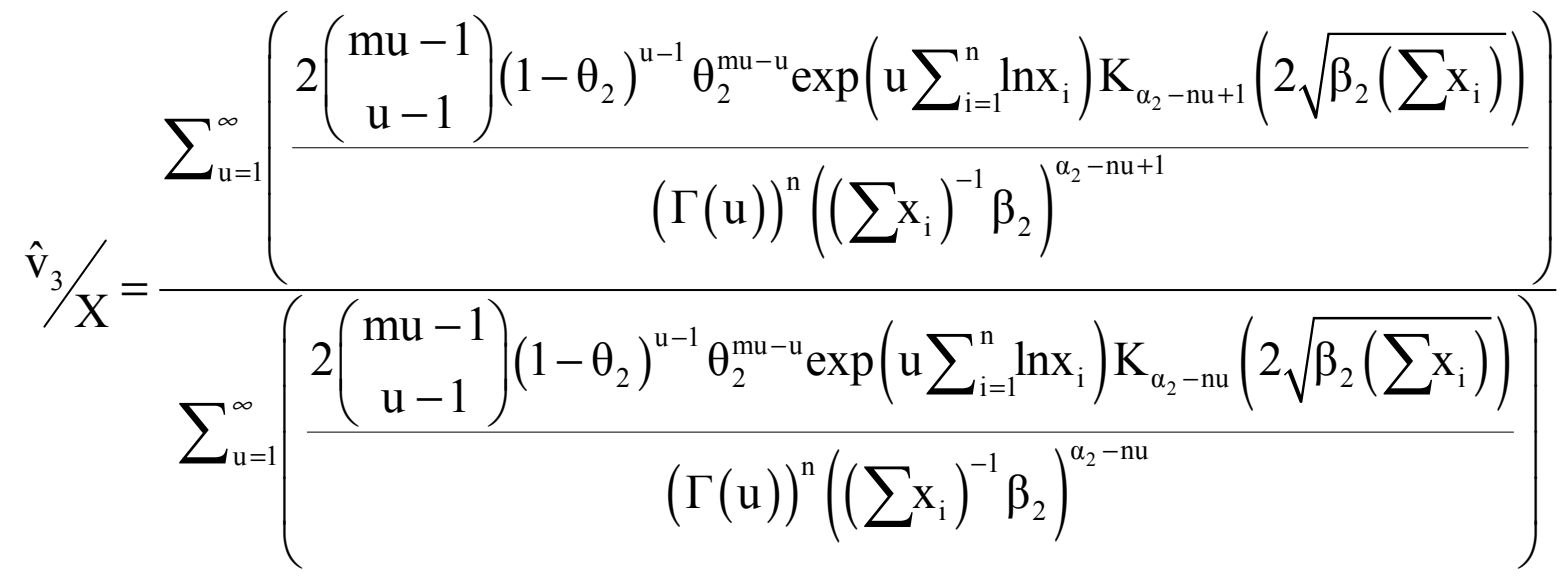




$$
\begin{aligned}
& \operatorname{Var}\left(\hat{\mathrm{u}}_{4} / \mathrm{X}\right)=\left(\frac{\sum_{\mathrm{u}=1}^{\infty}\left(\frac{2 \mathrm{u}^{2}\left(\begin{array}{c}
\mathrm{mu}-1 \\
\mathrm{u}-1
\end{array}\right)\left(1-\theta_{2}\right)^{\mathrm{u}-1} \theta_{2}^{\mathrm{mu}-\mathrm{u}} \exp \left(\mathrm{u} \sum_{\mathrm{i}=1}^{\mathrm{n}} \ln \mathrm{x}_{\mathrm{i}}\right) \mathrm{K}_{\mathrm{a}_{2}-\mathrm{nu}}\left(2 \sqrt{\beta_{2}\left(\sum \mathrm{x}_{\mathrm{i}}\right)}\right)}{(\Gamma(\mathrm{u}))^{\mathrm{n}}\left(\left(\sum \mathrm{x}_{\mathrm{i}}\right)^{-1} \beta_{2}\right)^{\alpha_{2}-\mathrm{nu}}}\right)}{\sum_{\mathrm{u}=1}^{\infty}\left(\frac{2\left(\begin{array}{c}
\mathrm{mu}-1 \\
\mathrm{u}-1
\end{array}\right)\left(1-\theta_{2}\right)^{\mathrm{u}-1} \theta_{2}^{\mathrm{mu}-\mathrm{u}} \exp \left(\mathrm{u} \sum_{\mathrm{i}=1}^{\mathrm{n}} \ln \mathrm{x}_{\mathrm{i}}\right) \mathrm{K}_{\mathrm{\alpha}_{2}-\mathrm{nu}}\left(2 \sqrt{\beta_{2}\left(\sum \mathrm{x}_{\mathrm{i}}\right)}\right)}{(\Gamma(\mathrm{u}))^{\mathrm{n}}\left(\left(\sum \mathrm{x}_{\mathrm{i}}\right)^{-1} \beta_{2}\right)^{\alpha_{2}-\mathrm{nu}}}\right)}\right) \\
& -\left(\frac{\sum_{\mathrm{u}=1}^{\infty}\left(\frac{2 \mathrm{u}\left(\begin{array}{c}
\mathrm{mu}-1 \\
\mathrm{u}-1
\end{array}\right)\left(1-\theta_{2}\right)^{\mathrm{u}-1} \theta_{2}^{\mathrm{mu}-\mathrm{u}} \exp \left(\mathrm{u} \sum_{\mathrm{i}=1}^{\mathrm{n}} \ln \mathrm{x}_{\mathrm{i}}\right) \mathrm{K}_{\alpha_{2}-\mathrm{nu}}\left(2 \sqrt{\beta_{2}\left(\sum \mathrm{x}_{\mathrm{i}}\right)}\right)}{(\Gamma(\mathrm{u}))^{\mathrm{n}}\left(\left(\sum \mathrm{x}_{\mathrm{i}}\right)^{-1} \beta_{2}\right)^{\alpha_{2}-\mathrm{nu}}}\right)}{\sum_{\mathrm{u}=1}^{\infty}\left(\frac{2\left(\begin{array}{c}
\mathrm{mu}-1 \\
\mathrm{u}-1
\end{array}\right)\left(1-\theta_{2}\right)^{\mathrm{u}-1} \theta_{2}^{\mathrm{mu}-\mathrm{u}} \exp \left(\mathrm{u} \sum_{\mathrm{i}=1}^{\mathrm{n}} \ln \mathrm{x}_{\mathrm{i}}\right) \mathrm{K}_{\alpha_{2}-\mathrm{nu}}\left(2 \sqrt{\beta_{2}\left(\sum \mathrm{x}_{\mathrm{i}}\right)}\right)}{(\Gamma(\mathrm{u}))^{\mathrm{n}}\left(\left(\sum \mathrm{x}_{\mathrm{i}}\right)^{-1} \beta_{2}\right)^{\alpha_{2}-\mathrm{nu}}}\right)}\right)^{2}
\end{aligned}
$$

$$
\begin{aligned}
& \operatorname{Var}\left(\hat{\mathrm{V}}_{4} / \mathrm{X}\right)=\frac{\sum_{\mathrm{u}=1}^{\infty}\left(\frac{2\left(\begin{array}{c}
\mathrm{mu}-1 \\
\mathrm{u}-1
\end{array}\right)\left(1-\theta_{2}\right)^{\mathrm{u}-1} \theta_{2}^{\mathrm{mu}-\mathrm{u}} \exp \left(\mathrm{u} \sum_{\mathrm{i}=1}^{\mathrm{n}} \ln \mathrm{x}_{\mathrm{i}}\right) \mathrm{K}_{\mathrm{a}_{2}-\mathrm{nu}+2}\left(2 \sqrt{\beta_{2}\left(\sum \mathrm{x}_{\mathrm{i}}\right)}\right)}{(\Gamma(\mathrm{u}))^{\mathrm{n}}\left(\left(\sum \mathrm{x}_{\mathrm{i}}\right)^{-1} \beta_{2}\right)^{\alpha_{2}-\mathrm{nu}+2}}\right)}{\sum_{\mathrm{u}=1}^{\infty}\left(\frac{2\left(\begin{array}{c}
\mathrm{mu}-1 \\
\mathrm{u}-1
\end{array}\right)\left(1-\theta_{2}\right)^{\mathrm{u}-1} \theta_{2}^{\mathrm{mu}-\mathrm{u}} \exp \left(\mathrm{u} \sum_{\mathrm{i}=1}^{\mathrm{n}} \ln \mathrm{x}_{\mathrm{i}}\right) \mathrm{K}_{\mathrm{\alpha}_{2}-\mathrm{nu}}\left(2 \sqrt{\beta_{2}\left(\sum \mathrm{x}_{\mathrm{i}}\right)}\right)}{(\Gamma(\mathrm{u}))^{\mathrm{n}}\left(\left(\sum \mathrm{x}_{\mathrm{i}}\right)^{-1} \beta_{2}\right)^{\alpha_{2}-\mathrm{nu}}}\right)} \\
& \left.-\left(\frac{\sum_{\mathrm{u}=1}^{\infty}\left(\frac{2\left(\begin{array}{c}
\mathrm{mu}-1 \\
\mathrm{u}-1
\end{array}\right)\left(1-\theta_{2}\right)^{\mathrm{u}-1} \theta_{2}^{\mathrm{mu}-\mathrm{u}} \exp \left(\mathrm{u} \sum_{\mathrm{i}=1}^{\mathrm{n}} \ln \mathrm{x}_{\mathrm{i}}\right) \mathrm{K}_{\alpha_{2}-\mathrm{nu}+1}\left(2 \sqrt{\beta_{2}\left(\sum \mathrm{x}_{\mathrm{i}}\right)}\right)}{(\Gamma(\mathrm{u}))^{\mathrm{n}}\left(\left(\sum \mathrm{x}_{\mathrm{i}}\right)^{-1} \beta_{2}\right)^{\alpha_{2}-\mathrm{nu}+1}}\right)}{\sum_{\mathrm{u}=1}^{\infty}\left(\frac{2\left(\begin{array}{c}
\mathrm{mu}-1 \\
\mathrm{u}-1
\end{array}\right)\left(1-\theta_{2}\right)^{\mathrm{u}-1} \theta_{2}^{\mathrm{mu}-\mathrm{u}} \exp \left(\mathrm{u} \sum_{\mathrm{i}=1}^{\mathrm{n}} \ln \mathrm{x}_{\mathrm{i}}\right) \mathrm{K}_{\alpha_{2}-\mathrm{nu}}\left(2 \sqrt{\beta_{2}\left(\sum \mathrm{x}_{\mathrm{i}}\right)}\right)}{(\Gamma(\mathrm{u}))^{\mathrm{n}}\left(\left(\sum \mathrm{x}_{\mathrm{i}}\right)^{-1} \beta_{2}\right)^{\alpha_{2}-\mathrm{nu}}}\right)}\right)\right)^{2}
\end{aligned}
$$




\section{BAYESIAN ESTIMATION OF ERLANG DISTRIBUTION UNDER DIFFERENT PRIORS}

Special Cases

For $\mathrm{m}=1$, the generalized truncated geometric distribution reduces to truncated geometric distribution, therefore, the Bayes estimator for scale parameter $\mathrm{u}$ and shape parameter $\mathrm{v}$ with their respective posterior variance is given by(3.2.4.11), (3.2.4.12), (3.2.4.13) and (3.2.4.14).

\section{References}

Bhattacharyya, S. K., \& Singh, N. K. (1994). Bayesian estimation of the traffic intensity in M/Ek/1 queue. Far. East, Journal of Math and Science, 2, 57-62.

Brockmeyer, E., Halstorm, H. L., \& Jenson, A. (1948). The life and works of A. K. Erlang. Transactions of the Danish Academy of Technical Sciences, 2, 277.
Damodaran, D., Gopal, G., \& Kapur, P. K. (2010). A Bayesian Erlang software reliability model. Communication in Dependability and Quality Management, 13(4), 82-90.

Erlang, A. K. (1909). The theory of probabilities and telephone conversations. Nyt Tidsskrift for Matematik B, 20(6), 87-98.

Evans, M., Hastings, N., \& Peacock, B. (2000). Statistical distributions, $3^{\text {rd }} E d$. New York, NY: John Wiley and Sons, Inc.

Haq, A., \& Dey, S. (2001). Bayesian estimation of Erlang distribution under different prior distributions. Journal of Reliability and Statistical Studies, 4(1), 1-30.

$$
\hat{\mathrm{u}}_{3}^{*} / \mathrm{X}=\frac{\sum_{\mathrm{u}=1}^{\infty}\left(\frac{2 \mathrm{u}\left(1-\theta_{2}\right)^{\mathrm{u}-1} \exp \left(\mathrm{u} \sum_{\mathrm{i}=1}^{\mathrm{n}} \ln \mathrm{x}_{\mathrm{i}}\right) \mathrm{K}_{\alpha_{2}-\mathrm{nu}}\left(2 \sqrt{\beta_{2}\left(\sum \mathrm{x}_{\mathrm{i}}\right)}\right)}{(\Gamma(\mathrm{u}))^{\mathrm{n}}\left(\left(\sum \mathrm{x}_{\mathrm{i}}\right)^{-1} \beta_{2}\right)^{\alpha_{2}-\mathrm{nu}}}\right)}{\sum_{\mathrm{u}=1}^{\infty}\left(\frac{2 \mathrm{u}\left(1-\theta_{2}\right)^{\mathrm{u}-1} \exp \left(\mathrm{u} \sum_{\mathrm{i}=1}^{\mathrm{n}} \ln \mathrm{x}_{\mathrm{i}}\right) \mathrm{K}_{\alpha_{2}-\mathrm{nu}}\left(2 \sqrt{\beta_{2}\left(\sum \mathrm{x}_{\mathrm{i}}\right)}\right)}{(\Gamma(\mathrm{u}))^{\mathrm{n}}\left(\left(\sum \mathrm{x}_{\mathrm{i}}\right)^{-1} \beta_{2}\right)^{\alpha_{2}-\mathrm{nu}}}\right)}
$$

$$
\hat{\mathrm{v}}_{3}^{*} / \mathrm{X}=\frac{\sum_{\mathrm{u}=1}^{\infty}\left(\frac{2\left(1-\theta_{2}\right)^{\mathrm{u}-1} \exp \left(\mathrm{u} \sum_{\mathrm{i}=1}^{\mathrm{n}} \ln \mathrm{x}_{\mathrm{i}}\right) \mathrm{K}_{\alpha_{2}-\mathrm{nu}+1}\left(2 \sqrt{\beta_{2}\left(\sum \mathrm{x}_{\mathrm{i}}\right)}\right)}{(\Gamma(\mathrm{u}))^{\mathrm{n}}\left(\left(\sum \mathrm{x}_{\mathrm{i}}\right)^{-1} \beta_{2}\right)^{\alpha_{2}-\mathrm{nu}+1}}\right)}{\sum_{\mathrm{u}=1}^{\infty}\left(\frac{2\left(1-\theta_{2}\right)^{\mathrm{u}-1} \exp \left(\mathrm{u} \sum_{\mathrm{i}=1}^{\mathrm{n}} \ln \mathrm{x}_{\mathrm{i}}\right) \mathrm{K}_{\alpha_{2}-\mathrm{nu}}\left(2 \sqrt{\beta_{2}\left(\sum \mathrm{x}_{\mathrm{i}}\right)}\right)}{(\Gamma(\mathrm{u}))^{\mathrm{n}}\left(\left(\sum \mathrm{x}_{\mathrm{i}}\right)^{-1} \beta_{2}\right)^{\alpha_{2}-\mathrm{nu}}}\right)}
$$




$$
\begin{aligned}
& \operatorname{Var}\left(\hat{\mathrm{u}}_{4}{ }^{*} / \mathrm{X}\right)=\left(\frac{\sum_{\mathrm{u}=1}^{\infty}\left(\frac{2 \mathrm{u}^{2}\left(1-\theta_{2}\right)^{\mathrm{u}-1} \exp \left(\mathrm{u} \sum_{\mathrm{i}=1}^{\mathrm{n}} \ln \mathrm{x}_{\mathrm{i}}\right) \mathrm{K}_{\alpha_{2}-\mathrm{nu}}\left(2 \sqrt{\beta_{2}\left(\sum \mathrm{x}_{\mathrm{i}}\right)}\right)}{(\Gamma(\mathrm{u}))^{\mathrm{n}}\left(\left(\sum \mathrm{x}_{\mathrm{i}}\right)^{-1} \beta_{2}\right)^{\alpha_{2}-\mathrm{nu}}}\right)}{\sum_{\mathrm{u}=1}^{\infty}\left(\frac{2 \mathrm{u}\left(1-\theta_{2}\right)^{\mathrm{u}-1} \exp \left(\mathrm{u} \sum_{\mathrm{i}=1}^{\mathrm{n}} \ln \mathrm{x}_{\mathrm{i}}\right) \mathrm{K}_{\alpha_{2}-\mathrm{nu}}\left(2 \sqrt{\beta_{2}\left(\sum \mathrm{x}_{\mathrm{i}}\right)}\right)}{(\Gamma(\mathrm{u}))^{\mathrm{n}}\left(\left(\sum \mathrm{x}_{\mathrm{i}}\right)^{-1} \beta_{2}\right)^{\alpha_{2}-\mathrm{nu}}}\right)}\right) \\
& -\left(\frac{\sum_{\mathrm{u}=1}^{\infty}\left(\frac{2 \mathrm{u}\left(1-\theta_{2}\right)^{\mathrm{u}-1} \exp \left(\mathrm{u} \sum_{\mathrm{i}=1}^{\mathrm{n}} \ln _{\mathrm{i}}\right) \mathrm{K}_{a_{2}-n u}\left(2 \sqrt{\beta_{2}\left(\sum \mathrm{x}_{\mathrm{i}}\right)}\right)}{(\Gamma(\mathrm{u}))^{\mathrm{n}}\left(\left(\sum \mathrm{x}_{\mathrm{i}}\right)^{-1} \beta_{2}\right)^{\alpha_{2}-\mathrm{nu}}}\right)}{\sum_{\mathrm{u}=1}^{\infty}\left(\frac{2 \mathrm{u}\left(1-\theta_{2}\right)^{\mathrm{u}-1} \exp \left(\mathrm{u} \sum_{\mathrm{i}=1}^{\mathrm{n}} \ln \mathrm{x}_{\mathrm{i}}\right) \mathrm{K}_{\mathrm{a}_{2}-\mathrm{nu}}\left(2 \sqrt{\beta_{2}\left(\sum \mathrm{x}_{\mathrm{i}}\right)}\right)}{(\Gamma(\mathrm{u}))^{\mathrm{n}}\left(\left(\sum \mathrm{x}_{\mathrm{i}}\right)^{-1} \beta_{2}\right)^{\alpha_{2}-\mathrm{nu}}}\right)}\right)^{2}
\end{aligned}
$$

$$
\begin{aligned}
& \operatorname{Var}\left(\hat{\mathrm{V}}_{4}^{*} / \mathrm{X}\right)=\left(\frac{\sum_{\mathrm{u}=1}^{\infty}\left(\frac{2\left(1-\theta_{2}\right)^{\mathrm{u}-1} \exp \left(\mathrm{u} \sum_{\mathrm{i}=1}^{\mathrm{n}} \ln \mathrm{x}_{\mathrm{i}}\right) \mathrm{K}_{\mathrm{a}_{2}-\mathrm{nu}+2}\left(2 \sqrt{\beta_{2}\left(\sum \mathrm{x}_{\mathrm{i}}\right)}\right)}{(\Gamma(\mathrm{u}))^{\mathrm{n}}\left(\left(\sum \mathrm{x}_{\mathrm{i}}\right)^{-1} \beta_{2}\right)^{\alpha_{2}-\mathrm{nu}+2}}\right)}{\sum_{\mathrm{u}=1}^{\infty}\left(\frac{2\left(1-\theta_{2}\right)^{\mathrm{u}-1} \exp \left(\mathrm{u} \sum_{\mathrm{i}=1}^{\mathrm{n}} \ln \mathrm{x}_{\mathrm{i}}\right) \mathrm{K}_{\mathrm{a}_{2}-\mathrm{nu}}\left(2 \sqrt{\beta_{2}\left(\sum \mathrm{x}_{\mathrm{i}}\right)}\right)}{(\Gamma(\mathrm{u}))^{\mathrm{n}}\left(\left(\sum \mathrm{x}_{\mathrm{i}}\right)^{-1} \beta_{2}\right)^{\alpha_{2}-\mathrm{nu}}}\right)}\right) \\
& -\left(\frac{\sum_{\mathrm{u}=1}^{\infty}\left(\frac{2\left(1-\theta_{2}\right)^{\mathrm{u}-1} \exp \left(\mathrm{u} \sum_{\mathrm{i}=1}^{\mathrm{n}} \ln _{\mathrm{i}}\right) \mathrm{K}_{\mathrm{a}_{2}-\mathrm{nu}+1}\left(2 \sqrt{\beta_{2}\left(\sum \mathrm{x}_{\mathrm{i}}\right)}\right)}{(\Gamma(\mathrm{u}))^{\mathrm{n}}\left(\left(\sum \mathrm{x}_{\mathrm{i}}\right)^{-1} \beta_{2}\right)^{\alpha_{2}-\mathrm{nu}+1}}\right)}{\sum_{\mathrm{u}=1}^{\infty}\left(\frac{2\left(1-\theta_{2}\right)^{\mathrm{u}-1} \exp \left(\mathrm{u} \sum_{\mathrm{i}=1}^{\mathrm{n}} \ln \mathrm{x}_{\mathrm{i}}\right) \mathrm{K}_{\mathrm{a}_{2}-\mathrm{nu}}\left(2 \sqrt{\beta_{2}\left(\sum \mathrm{x}_{\mathrm{i}}\right)}\right)}{(\Gamma(\mathrm{u}))^{\mathrm{n}}\left(\left(\sum \mathrm{x}_{\mathrm{i}}\right)^{-1} \beta_{2}\right)^{\alpha_{2}-\mathrm{nu}}}\right)}\right)^{2}
\end{aligned}
$$




\section{BAYESIAN ESTIMATION OF ERLANG DISTRIBUTION UNDER DIFFERENT PRIORS}

Harischandra, K., \& Rao, S. S. (1988). A note on statistical inference about the traffic intensity parameter in $\mathrm{M} / \mathrm{Ek} / 1$ queue. Sankhya, $B$, 50, 144-148.

Jain, S. (2001). Estimating the change point of Erlang interarrival time distribution. 39(2), 200-207: INFOR-OTTAWA- Technology Publications. University of Toronto Press, Canada.

Nair, U. N, Muraleedharan, G., \& Kurup, P. G. (2003). Erlang distribution model for ocean wave periods. Journal of the Indian Geophysical Union, 7(2), 59-70.
Suri, P. K, Bhushan, B., \& Jolly, A. (2009). Time estimation for project management life cycles: A simulation approach. International Journal of Computer Science and Network Security, 9(5), 211-215.

Wiper, M. P. (1998). Bayesian analysis of $\mathrm{Er} / \mathrm{M} / 1$ and $\mathrm{Er} / \mathrm{M} / \mathrm{C}$ queues. Journal of Statistical Planning and Inference, 69, 65-79.

Acknowledgements

The authors would like to thanks the referees for their careful reading of the manuscript and for their valuable comments and suggestions. 